\title{
A Characterization of Strategy-Proof Voting Rules for Separable Weak Orderings
}

\author{
Biung-Ghi Ju* \\ September 4, 2001 (Revision: January 25, 2003) \\ Forthcoming in Social Choice and Welfare
}

\begin{abstract}
We consider the problem of choosing a subset of a finite set of indivisible objects (public projects, facilities, laws, etc.) studied by Barberà, Sonnenschein, and Zhou (1991). Here we assume that agents' preferences are separable weak orderings. Given such a preference, objects are partitioned into three types, "goods", "bads", and "nulls". We focus on "voting rules", which rely only on this partition rather than the full information of preferences. We characterize voting rules satisfying strategy-proofness (no one can ever be better off by lying about his preference) and null-independence (the decision on each object should not be dependent on the preference of an agent for whom the object is a null). We also show that serially dictatorial rules are the only voting rules satisfying efficiency as well as the above two axioms. We show that the "separable domain" is the unique maximal domain over which each rule in the first characterization, satisfying a certain fairness property, is strategy-proof.
\end{abstract}

Keywords: Strategy-proofness, null-independence, efficiency, separable weak ordering

*Department of Economics, University of Kansas, 1300 Sunnyside Avenue, Lawrence, KS 66045-7585, USA. E-mail: bgju@ku.edu. Tel.: +1-(785)-864-2860. Fax.: +1-(785)-864-5270. I am grateful to Professor William Thomson for helpful comments and suggestions. I also thank Professor John Duggan, Christopher Chambers, and seminar participants in Department of Economics, University of Rochester. I thank anonymous referees for their detailed comments and suggestions that helped me a great deal to develop the earlier version of this paper. All remaining errors are mine. 


\section{Introduction}

Collective decision is often required to be made on multiple issues. We consider a simple model, in which each issue is associated with two possible decisions. For example, in Congress, legislators need to consider several bills at the same time. They can either accept or reject each bill. Another example is the problem of qualifying members of a society for a certain activity. Each member is either qualified or disqualified.

Such a problem can be represented by the problem of choosing a subset of a set of indivisible objects (public projects, facilities, laws, etc.) studied by Barberà, Sonnenschein, and Zhou (1991). ${ }^{1}$ Each subset is an alternative and agents have preferences over these alternatives. A social choice rule, or simply, a rule, associates with each preference profile a single desirable alternative. We are interested in rules satisfying strategy-proofness, the requirement that no one can ever be better off by lying about his preference.

When there is no restriction on admissible preferences, the Gibbard-Satterthwaite Theorem (Gibbard, 1973 and Satterthwaite, 1975) applies. Every strategy-proof rule satisfying the "full-range condition", or voter sovereignty, is dictatorial, when there are at least three alternatives. However, it is often the case that the consumption of an object affects agents' welfare separately from the consumptions of other objects. When preferences have such a "separability" restriction, the Gibbard-Satterthwaite Theorem does not apply. A great variety of strategy-proof rules, called schemes of "voting by committees", are characterized by Barberà, Sonnenschein, and Zhou (1991). They also show that within these rules, only dictatorial ones are efficient, when there are at least three objects. These results are established under the additional restriction of linearity (no indifference between any two alternatives) on preferences.

They do not apply directly in the domain of separable but possibly non-linear preferences, which we think is more natural. Even if non-linear preferences are admissible, all schemes of voting by committees are still strategy-proof. However, they may not be the only such rules. On the other hand, no scheme of voting by committees is efficient when non-linear preferences are admissible (dictatorial schemes are "weakly efficient" but not efficient). In order to satisfy efficiency, we need more sophisticated decisions. No earlier study on this choice problem, so far, provides a characterization of strategy-proof rules for separable and possibly non-linear preferences. We establish one such result by imposing the following two additional requirements.

\footnotetext{
${ }^{1}$ See also Barberà, Massó, and Neme $(1997,2000)$.
} 
Given a separable preference, objects are partitioned into three kinds; "goods", "bads", and "nulls" (an object is a good if its consumption always increases the welfare, independently of other objects; it is a bad if its consumption always decreases the welfare; it is a null if its consumption never makes any difference). ${ }^{2}$ We require that decisions should rely only on such simple "three-tiered" information rather than the full information of preferences. We refer to this requirement as votes-only property and refer to rules satisfying it as voting rules. We next introduce a new requirement, called null-independence, which says that if an object is a null for an agent, then his preference should not play a critical role in the decision on this object.

We characterize the family of strategy-proof rules satisfying votes-only property and null-independence. This family (to be explained later) includes all schemes of voting by committees; it is much bigger than that. Adding efficiency, we characterize "serially dictatorial rules", under which a priority ordering of agents is given and the first agent, the dictator, always guarantees one of his preferred alternatives and the second agent always guarantees one of his preferred alternatives in the set of dictator's preferred alternatives and so on. Even though we weaken efficiency to weak efficiency (no simultaneous welfare improvement of all agents is possible), we cannot escape dictatorship. Finally, we show that the separable domain is the unique maximal "rich" domain over which each rule in the first characterization, satisfying a certain fairness property, is strategy-proof.

Votes-only property coincides with "tops-only property" when preferences are linear. As shown by Barberà, Sonnenschein, and Zhou (1991), tops-only property is implied by strategy-proofness and voter sovereignty. However, when non-linear preferences are admissible, this implication does not hold: see Example ??. For linear preferences, any object is either a good or a bad (there is no null). So null-independence is vacuously satisfied by any rule.

Another important line of research on similar choice problems has been directed to study several normative requirements of social choice rules. Among others are Wilson (1975), Rubinstein and Fishburn (1986), Kasher and Rubinstein (1998), and Samet and Schmeidler (2001). ${ }^{3}$ We establish logical relations between our main requirements and the following two central requirements in

\footnotetext{
${ }^{2}$ When preferences are additively separable, an object is a good if and only if it gives positive utility, it is a bad if and only if it gives negative utility, and it is a null if and only if it gives zero utility.

${ }^{3}$ Kasher and Rubinstein (1998) and Samet and Schmeidler (2001) consider problems which are special examples of our choice problem. In particular, Samet and Schmeidler (2001) consider the "qualification problem", in which the set of objects coincide with the set of individuals.
} 
this literature. Monotonicity requires that when the set of goods expands and the set of bads contracts for each agent, the choice should expand. ${ }^{4}$ Independence requires that the decision on an object should rely only on agents' three-tiered evaluations on this object. ${ }^{5}$

We show that voting rules satisfying monotonicity and independence are described in terms of sets consisting of pairs of disjoint groups, called "power structures". For each object, a power structure is given and the object is chosen if and only if the group of agents in favor of the object and the group against it constitute a pair in that power structure. Each power structure has the natural property that the decisive power of a group against another increases if the former group expands and the latter group contracts. We call the family of all such rules Family $\Phi^{*}$. We show that a voting rule satisfies strategy-proofness and nullindependence if and only if it satisfies monotonicity and independence. Therefore, rules in Family $\Phi^{*}$ are the only voting rules satisfying strategy-proofness and null-independence. Adding some combinations of efficiency, voter sovereignty, anonymity, and neutrality, we characterize several subfamilies of Family $\Phi^{*}$.

Our study is related with the following literature. Public goods problems often have the form of decisions from a discrete or indiscrete box (Cartesian product of intervals). Components of each point in the box represent levels of their corresponding public goods provision. In the 1-dimensional case, Moulin (1980) exhibits a remarkable contrast to the Gibbard-Satterthwaite Theorem by characterizing a great variety of strategy-proof rules for "single-peaked preferences". He focuses on rules, called "voting schemes", which depend only on agents' peaks for each preference profile. He shows that a family of rules, called "generalized median voter schemes" are the only strategy-proof voting schemes that respect unanimity in the following sense; whenever everyone's peak coincides, the common peak should be chosen.

Border and Jordan (1983) strengthen Moulin's conclusion by characterizing the same family of rules in the 1-dimensional case without focusing on voting schemes. They, moreover, extend this conclusion to the multi-dimensional case. They consider several multi-dimensional counterparts of the 1-dimensional single-peakedness. "Star-shaped" preferences have the "1-dimensional single-

\footnotetext{
${ }^{4}$ Monotonicity is introduced by Kasher and Rubinstein (1998) and Samet and Schmeidler (2001) in slightly different forms. We generalize their notion to deal with non-linear preferences.

${ }^{5}$ Independence is introduced by Rubinstein and Fishburn (1986) in the abstract model of algebraic aggregation. Later, it is considered by Kasher and Rubinstein (1998) and Samet and Schmeidler (2001).
} 
peakedness" over each linear path through the peak. "Separable" preferences have a uniform "marginal preference" over each coordinate, independently of values of other coordinates. Over the domain of star-shaped separable preferences or the domain of quadratic separable preferences, they characterize strategy-proof rules that respect unanimity.

When the alternative space is a discrete box, Barberà, Gül, and Stacchetti (1993) consider linear preferences satisfying, what they call, "multi-dimensional singlepeakedness" (given an alternative $x$, any alternative in-between $x$ and the peak in terms of $L_{1}$-norm is ordered in-between $x$ and the peak). They show that "multidimensional generalized median voter schemes" are the only strategy-proof rules satisfying voter sovereignty. ${ }^{6}$ Their conclusion is generalized by Le Breton and Sen (1999). They identify a certain general richness condition for domains consisting of linear preferences. The condition encompasses a variety of domains including the domain of multi-dimensional single-peaked preferences. Under this richness condition, they characterize rules satisfying strategy-proofness and voter sovereignty. ${ }^{7}$ When each coordinate in the alternative space contains only two points, the domain of separable linear preferences satisfies the richness condition. Thus, their conclusion generalizes also the result by Barberà, Sonnenschein, and Zhou (1991), which is for such "coordinate-wise-binary choice problems".

Le Breton and Sen (1995) exhibit a difficulty of extending their "decomposability result" in linear preference domains to domains with possibly nonlinear preferences. In the coordinate-wise-binary case, we overcome such difficulty by imposing the two additional requirements, votes-only property and nullindependence, which do not have any logical relation with strategy-proofness and voter sovereignty altogether.

Le Breton and Weymark (1999) consider the multi-dimensional alternative space. While they also consider possibly non-linear preferences, their main conclusions rely either on the assumption that each coordinate has more than two points or on the assumption that each preference in the domain has a unique best alternative. Since neither of the two assumptions holds in our domain, their "component-wise dictatorship" conclusion does not apply. It is remarkable to notice that Le Breton and Weymark (1999) do not impose any additional requirement other than strategy-proofness and voter sovereignty. They prove that

\footnotetext{
${ }^{6}$ Voter sovereignty, here, can be replaced with the requirement of unanimity respect.

${ }^{7}$ More precisely, their richness is satisfied by a proper subdomain of the domain of multidimensional single-peaked preferences. They first characterize strategy-proof rules in this subdomain. Then they extend such characterization and obtains the result by Barberà, Gul, and Stacchetti (1993).
} 
strategy-proofness and voter sovereignty imply tops-only property over certain restricted domains that include possibly non-linear preferences but not all of them. When all non-linear preferences are admissible, such an implication does not hold.

The existence of normatively appealing strategy-proof rules over restricted domains casts the following important question. How far these domains can be enlarged with the possibility result intact? This question is addressed by Barberà, Sonnenschein, and Zhou (1991). They show that the separable linear domain is the unique maximal rich domain of linear preferences, over which normatively appealing schemes of voting by committees are strategy-proof. Whether a similar result holds without focusing on linear preferences is not studied by them. Since non-linear preferences may have multiple top alternatives, it is not clear how to define schemes of voting by committees. For separable preferences, such a difficulty can be resolved easily using the set of goods instead of top alternatives. In fact, the set of goods coincides with the intersection of all top alternatives for separable preferences. We make use of this intersection to define schemes of voting by committees for arbitrary domains and establish a similar maximal domain result without focusing on linear preferences. Our result also applies to other rules in Family $\Phi^{*}$ which are not schemes of voting by committees. Maximal domain results are also established in extended models of multidimensional problems by Serizawa (1995) and Le Breton and Sen (1999).

This paper is organized as follows. In Section 2, we introduce our model (Section 2.1) and define some important families of rules (Section 2.2). In Sec-

tion 3, we define our axioms. In Section 4, we state our characterization results (Section 4.1) and the maximal domain result (Section 4.2). We conclude with several remarks in Section 5. Finally, all proofs for Section 4.1 are collected in the Appendix.

\section{The model}

\subsection{Basic concepts}

There is a finite set $A$ of indivisible objects. Any subset of $A$ is an (social) alternative. There are $n \geq 2$ agents who have preferences (complete and transitive binary relations) over the set $2^{A}$ of all social alternatives. Let $N \equiv$ $\{1, \cdots, n\}$ be the set of all agents. For each preference $R_{i}$, we denote its strict relation by $P_{i}$ and indifference relation by $I_{i}$.

We focus on preferences with the following restriction. A preference $R_{i}$ is 
separable if for all $x \in A$ and all $X \subseteq A \backslash x,{ }^{8}$

$$
\begin{aligned}
& {[X \cup x] P_{i} X \text { if and only if } x P_{i} \varnothing ;} \\
& {[X \cup x] I_{i} X \text { if and only if } x I_{i} \varnothing .}
\end{aligned}
$$

Let $\mathcal{S}$ be the set of all separable preferences. A preference is additively separable if it has an additive numerical representation. Let $\mathcal{S}_{\boldsymbol{a d d}}$ be the set of all additively separable preferences. A preference is linear if no two alternatives are indifferent. Let $\mathcal{S}_{\text {lin }}$ be the set of all linear separable preferences. Note that when preferences are linear, the second part of the definition of separability holds vacuously. So in this case, our definition of separability coincides with the definition by Barberà, Sonnenschein, and Zhou (1991). For separable preferences, objects are partitioned into the following three kinds. An object $x \in A$ is a good for $\boldsymbol{R}_{\boldsymbol{i}} \in \mathcal{S}$ if for all $X \subseteq A \backslash x,[X \cup x] P_{i} X$. Object $x$ is a bad for $\boldsymbol{R}_{\boldsymbol{i}}$ if for all $X \subseteq A \backslash x, X P_{i}[X \cup x]$. Object $x$ is a null for $\boldsymbol{R}_{\boldsymbol{i}}$ if for all $X \subseteq A \backslash x, X$ $I_{i}[X \cup x]$. Let $\boldsymbol{G}\left(\boldsymbol{R}_{\boldsymbol{i}}\right)$ be the set of goods for $R_{i}$ and $\boldsymbol{B}\left(\boldsymbol{R}_{\boldsymbol{i}}\right)$ be the set of bads. For each $R \in \mathcal{S}^{N}$ and each $x \in A$, we say that $x$ is a null for $R$ when $x$ is a null for $R_{i}$ for each $i \in N{ }^{9}$ For all $R \in \mathcal{S}^{N}$ and all $x \in A, N$ is partitioned into the group of agents for whom $x$ is a good, denoted by $\boldsymbol{N}_{\boldsymbol{x}}^{\boldsymbol{G}}(\boldsymbol{R})$, the group of agents for whom $x$ is a bad, denoted by $\boldsymbol{N}_{\boldsymbol{x}}^{\boldsymbol{B}}(\boldsymbol{R})$, and the group of agents for whom $x$ is a null.

For each agent $i$, let $\mathcal{D}_{\boldsymbol{i}} \subseteq \mathcal{S}$ be a set of his admissible preferences. Let $\mathcal{D} \equiv \mathcal{D}_{1} \times \cdots \times \mathcal{D}_{n}$ be the set of profiles of admissible preferences. A social choice rule, or simply, a rule, is a function $\varphi: \mathcal{D} \rightarrow 2^{A}$ mapping each preference profile into a single alternative. Thus, we call $\mathcal{D}$ the domain of preference profiles. Important examples of domains are $\mathcal{S}^{N}, \mathcal{S}_{\text {add }}^{N}$, and $\mathcal{S}_{\text {lin }}^{N}$, which are called the separable domain, the additive domain, and the linear separable domain respectively.

We focus on rules that depend only on the simple information of preferences in terms of goods, bads, and nulls. Formally, a rule $\varphi$ is a voting rule if it satisfies the following property: for all $R, R^{\prime} \in \mathcal{D}$, if for all $i \in N, G\left(R_{i}\right)=G\left(R_{i}^{\prime}\right)$ and $B\left(R_{i}\right)=B\left(R_{i}^{\prime}\right)$, then $\varphi(R)=\varphi\left(R^{\prime}\right)$. We refer to this property as votes-only property. ${ }^{10}$

\footnotetext{
${ }^{8}$ For convenience, we denote each singleton $\{x\}$ by $x$.

${ }^{9}$ We use notation, $R, R^{\prime}, \bar{R}, \bar{R}^{\prime}$, etc. for elements in $\mathcal{R}^{N}$. Following standard notational convention, we write $i$ 's component of $R$ with $R_{i}$ and we write $i$ 's component of $R^{\prime}$ with $R_{i}^{\prime}$.

${ }^{10}$ In the domain of separable linear preferences, votes-only property coincides with the "topsonly property" in Barberà, Sonnenschein, and Zhou (1991).
} 


\section{Important Domain Properties}

Our main results are established over the separable domain $\mathcal{S}^{N}$ and they also hold over the additive domain $\mathcal{S}_{a d d}^{N}$. We specify several properties of the two domains, which will play critical roles. Our results will apply to any other subdomains of the separable domain satisfying these properties. Therefore, in stating the domain properties, we do not restrict our attention only to the two domains.

However, we are only interested in domains that admit a sufficiently large variety of preferences in the sense described by the following three properties A1, A2, and A3.

The first property is that each object is potentially a good and also potentially a bad in the domain.

A1. For all $i \in N$ and all $x \in A$, there exists $R_{i}, R_{i}^{\prime} \in \mathcal{D}_{i}$ such that $x \in G\left(R_{i}\right)$ and $x \in B\left(R_{i}^{\prime}\right)$.

The second property is that for each agent $i$ and each object $x$, if $x$ is not a good for a preference $R_{i}$, then there is another admissible preference $R_{i}^{\prime}$ that has more goods, including $x$, and less bads than $R_{i}$.

A2. For all $i \in N$, all $R_{i} \in \mathcal{D}_{i}$ and all $x \in A$ with $x \notin G\left(R_{i}\right)$, there exists $R_{i}^{\prime} \in \mathcal{D}_{i}$ such that $G\left(R_{i}^{\prime}\right) \supseteq G\left(R_{i}\right) \cup x$ and $B\left(R_{i}^{\prime}\right) \subseteq B\left(R_{i}\right) \backslash x$.

The third property is that for each agent $i$ and each object $x$, if $x$ is a bad and a null for preferences $R_{i}$ and $R_{i}^{\prime}$, respectively, then there is another admissible preference $R_{i}^{\prime \prime}$ that has more goods and less bads than $R_{i}$ and for which $x$ is a null.

A3. For all $i \in N$ and all $R_{i}, R_{i}^{\prime} \in \mathcal{D}_{i}$ with $x \in B\left(R_{i}\right)$ and $x \notin G\left(R_{i}^{\prime}\right) \cup B\left(R_{i}^{\prime}\right)$, there exists $R_{i}^{\prime \prime} \in \mathcal{D}_{i}$ such that $G\left(R_{i}^{\prime \prime}\right) \supseteq G\left(R_{i}\right), B\left(R_{i}^{\prime \prime}\right) \subseteq B\left(R_{i}\right)$, and $x \notin$ $G\left(R_{i}^{\prime \prime}\right) \cup B\left(R_{i}^{\prime \prime}\right)$.

Note that A3 is trivially satisfied by any domain in which no preference has any null; for example, the linear separable domain $\mathcal{S}_{\text {lin }}^{N}$ and any subdomain of $\mathcal{S}_{\text {lin }}^{N}$. Two properties, A2 and A3, are somewhat complicated. However, they are implied by the following natural property. It states that for all two disjoint subsets of objects, each agent has an admissible preference in which the two sets become the set of goods and the set of bads. 
A4. For all $i \in N$ and all disjoint $X, X^{\prime} \subseteq A$, there exists $R_{i} \in \mathcal{D}_{i}$ such that $G\left(R_{i}\right)=X$ and $B\left(R_{i}\right)=X^{\prime}{ }^{11}$

Note that A4 is not satisfied by the linear separable domain $\mathcal{S}_{\text {lin }}^{N}$. Yet, both A2 and A3 are satisfied by $\mathcal{S}_{\text {lin }}^{N}$. Property A4 is similar to the "richness property" by Barberà, Sonnenschein, and Zhou (1991, p.605). Their richness property is stronger than the combination of A1, A2, and A3, for the case of linear separable preferences. $^{12}$ The three properties are crucial for showing Proposition 1. We take several examples of domains satisfying them.

Example 1. Domains satisfying A1, A2, and A3.

(i) The separable domain $\mathcal{S}^{N}$, the additive domain $\mathcal{S}_{\text {add }}^{N}$, and the linear separable domain $\mathcal{S}_{\text {lin }}^{N}$ satisfy A1-A3.

(ii) A preference $R_{0} \in \mathcal{S}$ is trichotomous if it is described by goods, bads, and nulls as follows: for all $X, X^{\prime} \subseteq A$,

$X R_{0} X^{\prime}$ if and only if $\left|G\left(R_{0}\right) \cap X\right|-\left|B\left(R_{0}\right) \cap X\right| \geq\left|G\left(R_{0}\right) \cap X^{\prime}\right|-\left|B\left(R_{0}\right) \cap X^{\prime}\right|$. Thus any agent with a trichotomous preference cares only about the "net" number of goods (the number of goods minus the number of bads). Clearly, each trichotomous preference is additive. Also all goods are indifferent, all bads are indifferent, and the utility of each good is equal to the disutility of each bad. Let $\mathcal{S}_{\text {Tri }}$ be the family of all trichotomous preferences. It is easy to show that $\mathcal{S}_{\text {Tri }}^{N}$ satisfies A1-A3.

(iii) A preference $R_{0} \in \mathcal{S}$ is dichotomous if it is trichotomous and all objects are either goods or bads (no null). Let $\mathcal{S}_{\mathrm{Di}}$ be the family of all dichotomous preferences. By definition, $\mathcal{S}_{\text {Tri }} \supset \mathcal{S}_{\text {Di }}$. It is easy to show that $\mathcal{S}_{\text {Di }}^{N}$ satisfies A1-A3.

We will make use of the following additional properties of the separable domain $\mathcal{S}^{N}$ and the additive domain $\mathcal{S}_{\text {add }}^{N}$. Roughly speaking, both of the first two properties state that there are at least three objects and the domain admits a sufficient variety of preferences in ordering the three objects.

Definition. A domain $\mathcal{D}$ satisfies Property $\boldsymbol{P}$ if there exist at least three objects and $\mathcal{D}$ satisfies the following two conditions:

P1. For all $i \in N$ and all distinct $x, y, z \in A$, there exist $R_{i}, R_{i}^{\prime}, \bar{R}_{i}, \bar{R}_{i}^{\prime} \in \mathcal{D}_{i}$ such that $x P_{i}\{x, z\} P_{i}$ y $P_{i} \varnothing P_{i} z$ and for all $w \notin\{x, y, z\}, \varnothing P_{i} w$;

$x P_{i}^{\prime} \varnothing P_{i}^{\prime} y P_{i}^{\prime}\{x, z\} P_{i}^{\prime} z$ and for all $w \notin\{x, y, z\}, \varnothing P_{i}^{\prime} w$;

$x \bar{P}_{i} \varnothing, y \bar{P}_{i} \varnothing, z \bar{P}_{i}\{x, y\}$, and for all $w \notin\{x, y, z\}, \varnothing \bar{P}_{i} w$;

\footnotetext{
${ }^{11} \mathrm{It}$ is easy to show that this property implies A2 and A3. For A2, let $X \equiv G\left(R_{i}\right) \cup x$ and $X^{\prime} \equiv B\left(R_{i}\right) \backslash x$. For $\mathrm{A} 3$, let $X \equiv G\left(R_{i}\right)$ and $X^{\prime} \equiv B\left(R_{i}\right) \backslash x$.

${ }^{12}$ The proof is available under request.
} 
$\varnothing \bar{P}_{i}^{\prime} x, \varnothing \bar{P}_{i}^{\prime} y,\{x, y\} \bar{P}_{i}^{\prime} z$, and for all $w \notin\{x, y, z\}, \varnothing \bar{P}_{i}^{\prime} w$.

P2. For all $i \in N$ and all distinct $x, y, z \in A$, there exist $R_{i}, R_{i}^{\prime} \in \mathcal{D}_{i}$ such that

$x I_{i} y I_{i} \varnothing$ and for all $w \notin\{x, y\}, \varnothing P_{i} w$;

$x I_{i}^{\prime} y I_{i}^{\prime} z I_{i}^{\prime} \varnothing$ and for all $w \notin\{x, y, z\}, \varnothing P_{i}^{\prime} w$.

Definition. A domain $\mathcal{D}$ satisfies Property $\boldsymbol{Q}$ if there exist at least three objects and $\mathcal{D}$ satisfies the following two conditions:

Q1. For all $i \in N$ and all distinct $x, y, z \in A$, there exist $R_{i}, R_{i}^{\prime}, \bar{R}_{i}, \bar{R}_{i}^{\prime} \in \mathcal{D}_{i}$ such that $y P_{i} \varnothing P_{i} z$ and for all $Y, Y^{\prime} \subseteq A \backslash x,[Y \cup x] P_{i} Y^{\prime}$;

$x P_{i}^{\prime} \varnothing P_{i}^{\prime} y$ and for all $Y, Y^{\prime} \subseteq A \backslash z, Y P_{i}^{\prime}\left[Y^{\prime} \cup z\right]$;

$x \bar{P}_{i} \varnothing, y \bar{P}_{i} \varnothing$, and for all $Y, Y^{\prime} \subseteq A \backslash z,[Y \cup z] \bar{P}_{i} Y^{\prime}$;

$\varnothing \bar{P}_{i}^{\prime} x, \varnothing \bar{P}_{i}^{\prime} y$, and for all $Y, Y^{\prime} \subseteq A \backslash z, Y \bar{P}_{i}^{\prime}\left[Y^{\prime} \cup z\right]$.

Q2. For all $i \in N$ and all distinct $x, y, z \in A$, there exists $R_{i} \in \mathcal{D}_{i}$ such that $x I_{i} y I_{i} z I_{i} \varnothing$.

Either one of Property $\mathrm{P}$ and Property Q is crucial for showing Propositions 2 and 3 .

The next property says that for any preference and any good $x$ (bad, respectively), there is an admissible preference that has the same sets of goods and bads as the initial preference and in which the utility (disutility, respectively) of $x$ is so great that having $x$ is always better (worse, respectively) than not having it, independently of decisions on other objects.

Definition. A domain $\mathcal{D}$ satisfies Property $\boldsymbol{R}$ if $\mathcal{D}$ satisfies the following two conditions:

R1. For all $i \in N$, all $R_{i} \in \mathcal{D}_{i}$, and all $x \in G\left(R_{i}\right)$, there exists $R_{i}^{\prime} \in \mathcal{D}_{i}$ such that $G\left(R_{i}^{\prime}\right)=G\left(R_{i}\right), B\left(R_{i}^{\prime}\right)=B\left(R_{i}\right)$, and for all $Y, Y^{\prime} \subseteq A \backslash x,[Y \cup x] P_{i}^{\prime} Y^{\prime}$.

R2. For all $i \in N$, all $R_{i} \in \mathcal{D}_{i}$, and all $x \in B\left(R_{i}\right)$, there exists $R_{i}^{\prime} \in \mathcal{D}_{i}$ such that $G\left(R_{i}^{\prime}\right)=G\left(R_{i}\right), B\left(R_{i}^{\prime}\right)=B\left(R_{i}\right)$, and for all $Y, Y^{\prime} \subseteq A \backslash x, Y P_{i}^{\prime}\left[Y^{\prime} \cup x\right]$.

Property $\mathrm{R}$ is crucial for showing Proposition 4 and Theorems 1 and 2. Note that the linear separable domain $\mathcal{S}_{\text {lin }}^{N}$ violates Properties $\mathrm{P}$ and $\mathrm{Q}$ but satisfies

Property R. Neither the trichotomous domain $\mathcal{S}_{\text {Tri }}^{N}$ nor the dichotomous domain $\mathcal{S}_{\mathrm{Di}}^{N}$ in Example 1 satisfies any of the three properties.

\subsection{Examples of voting rules}

We define an important family of voting rules, crucial in our work. These rules make the decision on each object, according to a predetermined set of ordered pairs of disjoint groups, or a "power structure", as follows. The ob- 
ject is chosen if and only if the group of agents in favor of the object and the group of agents against the object constitute a pair in that set. In this case, the unanimous approval of the object by members in the first group "overpowers" the unanimous objection by members in the second group. Formally, let $\mathfrak{C}^{*} \equiv\left\{\left(C_{1}, C_{2}\right) \in 2^{N} \times 2^{N}: C_{1} \cap C_{2}=\varnothing\right\}$ be the set of all pairs of disjoint groups of agents. For each $x \in A$, let $\mathfrak{C}_{x} \subseteq \mathfrak{C}^{*}$. When $\left(C_{1}, C_{2}\right) \in \mathfrak{C}_{x}$, we say that $C_{1}$ overpowers $C_{2}$. Set $\mathfrak{C}_{x}$ satisfies power-monotonicity, or $\boldsymbol{P}$-monotonicity if whenever group $C_{1}$ overpowers group $C_{2}$, each supergroup of $C_{1}$ also overpowers each disjoint subgroup of $C_{2}$ : that is, for all $\left(C_{1}, C_{2}\right) \in \mathfrak{C}_{x}$, if $\left(C_{1}^{\prime}, C_{2}^{\prime}\right) \in \mathfrak{C}^{*}$ is such that $C_{1}^{\prime} \supseteq C_{1}$ and $C_{2}^{\prime} \subseteq C_{2}$, then $\left(C_{1}^{\prime}, C_{2}^{\prime}\right) \in \mathfrak{C}_{x}$. For each $x \in A$, a power structure associated with $\boldsymbol{x}$ is a set $\mathfrak{C}_{x}$ of pairs of disjoint groups, satisfying P-monotonicity. A profile of power structures is a list $\left(\mathfrak{C}_{x}\right)_{x \in A}$ of power structures indexed by objects.

Definition. A rule is in Family $\boldsymbol{\Phi}^{*}$ if there exists a profile of power structures $\left(\mathfrak{C}_{x}\right)_{x \in A}$ such that for all $R \in \mathcal{D}$ and all $x \in A, x \in \varphi(R)$ if and only if $\left(N_{x}^{G}(R), N_{x}^{B}(R)\right) \in \mathfrak{C}_{x} \cdot{ }^{13}$

Several properties of profiles of power structures are in order. A profile $\left(\mathfrak{C}_{x}\right)_{x \in A}$ satisfies power-unanimity, or $\boldsymbol{P}$-unanimity if for all $x \in A,(N, \varnothing) \in \mathfrak{C}_{x}$ and $(\varnothing, N) \notin \mathfrak{C}_{x}$. It satisfies power-neutrality, or $\boldsymbol{P}$-neutrality if for all $x, y \in A$ and all $\left(C_{1}, C_{2}\right) \in \mathfrak{C}^{*},\left(C_{1}, C_{2}\right) \in \mathfrak{C}_{x} \Leftrightarrow\left(C_{1}, C_{2}\right) \in \mathfrak{C}_{y}$. It satisfies power-anonymity, or $\boldsymbol{P}$-anonymity if for each $x \in A, \mathfrak{C}_{x}$ can be described by a set $\mathcal{I}_{x} \subseteq\left\{\left(t_{1}, t_{2}\right) \in\right.$ $\left.\mathbb{Z}_{+} \times \mathbb{Z}_{+}: t_{1}+t_{2} \leq n\right\}$ as follows: $\left(C_{1}, C_{2}\right) \in \mathfrak{C}_{x} \Leftrightarrow\left(\left|C_{1}\right|,\left|C_{2}\right|\right) \in \mathcal{I}_{x} \cdot{ }^{14}$ We call $\mathcal{I}_{x}$ the index set for $\boldsymbol{x}$.

Definition (Barberà, Sonnenschein, and Zhou, 1991). A rule $\varphi$ is a scheme of voting by committees if for each $x \in A$, there exists a nonempty collection $\mathcal{C}_{x}$ of groups of agents such that (i) $\varnothing \notin \mathcal{C}_{x}$; (ii) for all $C_{0} \in \mathcal{C}_{x}$ and all $C_{0}^{\prime} \supseteq C_{0}$, $C_{0}^{\prime} \in \mathcal{C}_{x}$; (iii) for all $R \in \mathcal{D}, x \in \varphi(R) \Leftrightarrow N_{x}^{G}(R) \in \mathcal{C}_{x}$.

Any scheme of voting by committees associated with $\left(\mathcal{C}_{x}\right)_{x \in A}$ is the rule in Family $\Phi^{*}$ associated with the following profile of simple power structures $\left(\mathfrak{C}_{x}\right)_{x \in A}$. For all $x \in A$, let $\mathfrak{C}_{x} \equiv\left\{\left(C_{1}, C_{2}\right) \in \mathfrak{C}^{*}: C_{1} \in \mathcal{C}_{x}, C_{2} \subseteq N \backslash C_{1}\right\}$. Note that by nonemptiness of $\mathcal{C}_{x}, \mathfrak{C}_{x}$ is nonempty. Then by part (i) in the above definition,

\footnotetext{
${ }^{13}$ Power structures and Family $\Phi^{*}$ are similar to "binary constitutions" and "binary decision rules" by Ferejohn and Fishburn (1979), which are preference aggregation rules in the Arrovian social choice model. Binary constitutions have another restriction, called "asymmetry", since they are used to determine social ordering of two alternatives.

${ }^{14}$ We denote the set of non-negative integers by $\mathbb{Z}_{+}$.
} 
the profile satisfies P-unanimity. Hence over the linear separable domain $\mathcal{S}_{\text {lin }}^{N}$ and also over any subdomain of $\mathcal{S}_{\text {lin }}^{N}$, the family of schemes of voting by committees coincide with the subfamily of Family $\Phi^{*}$, consisting of rules associated with P-unanimous profile of power structures.

For all $i \in N$, all $R_{i} \in \mathcal{D}_{i}$, and all $\mathcal{X} \subseteq 2^{A}$, let $\operatorname{Max}\left[R_{i}: \mathcal{X}\right]$ be the set of all best alternatives for $R_{i}$ in $\mathcal{X}$. A rule $\varphi$ is dictatorial if there exists $i \in N$ such that for all $R \in \mathcal{D}, \varphi(R) \in \operatorname{Max}\left[R_{i}: 2^{A}\right]$. We call such $i$ the dictator. Not every dictatorial rule is in Family $\Phi^{*}$. A rule in Family $\Phi^{*}$ is dictatorial if and only if its profile of power structures $\left(\mathfrak{C}_{x}\right)_{x \in A}$ has the following property: there exists $i \in N$ such that for all $x \in A,(i, N \backslash i) \in \mathfrak{C}_{x}$ and $(N \backslash i, i) \notin \mathfrak{C}_{x}$.

Let $\pi$ be a permutation on $N$. Let $R \in \mathcal{D}$. Let $M^{1}(R, \pi) \equiv \operatorname{Max}\left[R_{\pi(1)}: 2^{A}\right]$. For all $k \in\{2, \cdots, n\}$, let $M^{k}(R, \pi) \equiv \operatorname{Max}\left[R_{\pi(k)}: M^{k-1}(R, \pi)\right]$. A rule $\varphi$ is serially dictatorial with respect to $\boldsymbol{\pi}$ if for all $R \in \mathcal{D}$ and all $k \in N$, $\varphi(R) \in M^{k}(R, \pi)$. Not every serially dictatorial rule is in Family $\Phi^{*}$. The power structure $\left(\mathfrak{C}_{x}\right)_{x \in A}$ of the serially dictatorial rule in Family $\Phi^{*}$, associated with $\pi$ is such that for all $x \in A$ and all disjoint non-empty groups $C_{1}, C_{2} \subseteq N$, $\left(C_{1}, C_{2}\right) \in \mathfrak{C}_{x}$ if and only if there exists $k \in\{1, \cdots, n\}$ such that $\pi(k) \in C_{1}$ and for all $k^{\prime}<k, \pi\left(k^{\prime}\right) \notin C_{1} \cup C_{2}$.

Family $\Phi^{*}$ is a subset of a larger family of voting rules, in which decisions are made in the following procedure. First, for each object, the set of agents in favor of it and the set of agents against it are identified. Second, based on these two groups, the "score" for the object is determined by a "scoring function" that maps each pair of disjoint groups into the score (a real number) for the object. Third, given a list of scores for all objects, the "threshold score" is determined by a "threshold scoring function" that maps each list of scores into a threshold score. Finally, each object is accepted if and only if its score is higher than or equal to the threshold score. Formally, a scoring function associated with $\boldsymbol{x}$ is a mapping $s_{x}: \mathfrak{C}^{*} \rightarrow \mathbb{R}$ satisfying the following property: for all $\left(C_{1}, C_{2}\right),\left(C_{1}^{\prime}, C_{2}^{\prime}\right) \in \mathfrak{C}^{*}$, if $C_{1} \subseteq C_{1}^{\prime}$ and $C_{2} \supseteq C_{2}^{\prime}$, then $s_{x}\left(C_{1}, C_{2}\right) \leq s_{x}\left(C_{1}^{\prime}, C_{2}^{\prime}\right)$. A threshold scoring function is a mapping $\bar{s}: \mathbb{R}^{|A|} \rightarrow \mathbb{R}$ satisfying the following property: for all $\left(S_{x}\right)_{x \in A},\left(S_{x}^{\prime}\right)_{x \in A} \in \mathbb{R}^{|A|}$, if $\left(S_{x}\right)_{x \in A} \leq\left(S_{x}^{\prime}\right)_{x \in A}$, then $\bar{s}\left(\left(S_{x}\right)_{x \in A}\right) \leq \bar{s}\left(\left(S_{x}^{\prime}\right)_{x \in A}\right)$. A rule $\varphi$ is a scoring rule if for each $x \in A$, there exist a scoring function $s_{x}$ and a threshold scoring function $\bar{s}$ such that for all $R \in \mathcal{D}$ and all $x \in A$,

$$
x \in \varphi(R) \Leftrightarrow s_{x}\left(N_{x}^{G}(R), N_{x}^{B}(R)\right) \geq \bar{s}\left(\left(s_{x}\left(N_{x}^{G}(R), N_{x}^{B}(R)\right)\right)_{x \in A}\right) .
$$

For example, plurality-based scoring rule, denoted by $\varphi^{\text {pl-scoring }}$, is the scoring rule that is associated with the following scoring functions $\left(s_{x}\right)_{x \in A}$ and threshold scoring function $\bar{s}$ : for each $x \in A$ and each $\left(C_{1}, C_{2}\right) \in \mathfrak{C}^{*}, s_{x}\left(C_{1}, C_{2}\right) \equiv$ 
$\left|C_{1}\right|-\left|C_{2}\right|-1$; for each $\left(S_{x}\right)_{x \in A} \in \mathbb{R}^{|A|}, \bar{s}\left(\left(S_{x}\right)_{x \in A}\right) \equiv \max \left\{\sum_{x \in A} S_{x} /|A|, 0\right\}$. Clearly, if an object is accepted by plurality-based scoring rule, then there are more agents in favor of it than agents against it.

Every rule in Family $\Phi^{*}$, associated with $\left(\mathfrak{C}_{x}\right)_{x \in A}$, is a scoring rule whose threshold scoring function is constant with the value 1 and whose profile of scoring functions $\left(s_{x}\right)_{x \in A}$ are such that for all $x \in A$ and all $\left(C_{1}, C_{2}\right) \in \mathfrak{C}^{*}$,

$$
s_{x}\left(C_{1}, C_{2}\right)=\left\{\begin{array}{l}
1, \text { if }\left(C_{1}, C_{2}\right) \in \mathfrak{C}_{x} ; \\
0, \text { otherwise }
\end{array}\right.
$$

Note that for any scoring rule, the score for each object $x$ at a preference profile $R$ is determined by $\left(N_{x}^{G}(R), N_{x}^{B}(R)\right)$ and so other information in $\left(G\left(R_{i}\right), B\left(R_{i}\right)\right)_{i \in N}$ do not play any role for the score of $x$. In particular, for rules in Family $\Phi^{*}$, the decision on $x$, not just the score, rely only on $\left(N_{x}^{G}(R), N_{x}^{B}(R)\right)$; decisions on objects are made "independently". However there are a variety of other types of voting rules. For example, weighted plurality rule, denoted by $\varphi^{\text {weight-pl, }}$ is the voting rule such that for all $R \in \mathcal{D}$ and all $x \in A, x \in \varphi^{\text {weight-pl }}(R) \Leftrightarrow$ $\sum_{i \in N_{x}^{G}(R)} \frac{1}{\left|G\left(R_{i}\right)\right|}>\sum_{i \in N_{x}^{B}(R)} \frac{1}{\left|B\left(R_{i}\right)\right|}$. Thus, for the decision on $x$, the positive vote by an agent $i$ in favor of $x$ is weighted by $\frac{1}{\left|G\left(R_{i}\right)\right|}$ and the negative vote by an agent $i$ against $x$ is weighted by $\frac{1}{\mid B\left(R_{i}\right)} ; x$ is accepted if and only if the sum of weighted positive votes is bigger than the sum of weighted negative votes.

\section{Axioms}

In this section, we define several important strategic and non-strategic requirements, or axioms, of rules. We start with our two main axioms. The first axiom requires that misrepresenting one's preference should never pay off, independently of what others' representations are.

Strategy-Proofness. For all $R \in \mathcal{D}$, all $i \in N$, and all $R_{i}^{\prime} \in \mathcal{D}_{i}$,

$$
\varphi\left(R_{i}, R_{-i}\right) R_{i} \varphi\left(R_{i}^{\prime}, R_{-i}\right) .
$$

If a rule satisfies strategy-proofness, it can be implemented in dominant strategy equilibrium in the "direct revelation mechanism", and conversely. We refer readers to Gibbard (1973), Satterthwaite (1975), Barberà, Sonnenschein, and Zhou (1991), and Thomson (2000) for more discussions on strategy-proofness. In particular, Barberà, Sonnenschein, and Zhou (1991) consider the same model as ours and study strategy-proof rules over the linear separable domain. 
We next introduce a new axiom. Suppose that an object $a$ is a null for an agent $i$. Then the decision on $a$ will never have any effect on agent $i$ 's welfare and so he will be indifferent to the decision. It would be unappealing if the preference of such an agent plays a key role in the decision on $a$. The next axiom rules out this possibility. Formally:

Null-Independence. For all $i \in N$, all $x \in A$, all $R_{i}, R_{i}^{\prime} \in \mathcal{D}_{i}$, and all $R_{-i} \in$ $\Pi_{j \neq i} \mathcal{D}_{j}$, if $x$ is null for both $R_{i}$ and $R_{i}^{\prime}$, then

$$
x \in \varphi\left(R_{i}, R_{-i}\right) \Leftrightarrow x \in \varphi\left(R_{i}^{\prime}, R_{-i}\right)
$$

Example 2. Family $\Phi^{*}$. Every rule in Family $\Phi^{*}$ satisfies both axioms. Nullindependence is trivial. Strategy-proofness can be shown as follows. Each rule $\varphi$ in Family $\Phi^{*}$ makes decisions object-by-object. That is, the decision on each object $x$ relies only on the group of agents in favor of $x$ and the group of agents against $x$. Moreover, the decision responds non-negatively to the increase in the first group and non-positively to the increase in the second group. Thus, when an agent is in favor of $x$, he cannot increase the chance of the acceptance of $x$ by saying he is against $x$. He cannot be better off by such a lie. Similarly, an agent cannot be better off by saying he is in favor of $x$, when he is against $x$. Therefore truthful announcement is always weakly better than lying, independently of what others' announcements are.

Example 3. Scoring rules. Our results will imply that all scoring rules not in Family $\Phi^{*}$ violate at least one of the two axioms. Here we show that pluralitybased scoring rule $\varphi^{\text {pl-scoring }}$ violates both strategy-proofness and null-independence. For simplicity, suppose that there are three agents and three objects and let $N \equiv\{1,2,3\}$ and $A \equiv\{a, b, c\}$. Consider a preference profile $\left(R_{1}, R_{2}, R_{3}\right)$ such that $\left(G\left(R_{1}\right), B\left(R_{1}\right)\right)=(\{a, b\}, \varnothing),\left(G\left(R_{2}\right), B\left(R_{2}\right)\right)=(\{a, c\},\{b\})$, and $\left(G\left(R_{3}\right), B\left(R_{3}\right)\right)=(\{a, b\},\{c\})$. Then the score of $a$ is 2 , the score of $b$ is 0 and the score of $c$ is -1 . Therefore the threshold score is the average score $1 / 3$ and so only object $a$ is chosen, that is, $\varphi^{\text {pl-scoring }}(R)=\{a\}$. Now suppose that agent 1 with true preference $R_{1}$ reports $R_{1}^{\prime}$ such that $\left(G\left(R_{1}^{\prime}\right), B\left(R_{1}^{\prime}\right)\right)=(\{a, b\},\{c\})$. Then the score of $c$ decreases to -2 , which lowers the average score and the threshold score to 0 . Since there is no change in the scores of $a$ and $b, a$ is still chosen and now, in addition, $b$ is chosen, that is, $\varphi^{\text {pl-scoring }}\left(R_{1}^{\prime}, R_{2}, R_{3}\right)=\{a, b\}$. Since $\{a, b\} P_{1}$ $\{a\}$, agent 1 is better off after the misrepresentation with $R_{1}^{\prime}$. It is easy to show that this rule also violates null-independence. ${ }^{15}$

${ }^{15}$ Let $R_{1}, R_{2}$, and $R_{3}$ be such that $\left(G\left(R_{1}\right), B\left(R_{1}\right)\right)=(\{a, b\}, \varnothing),\left(G\left(R_{2}\right), B\left(R_{2}\right)\right)=$ 
We relate the two main axioms with the following two axioms, called "monotonicity" and "independence", studied by Rubinstein and Fishburn (1986), Kasher and Rubinstein (1998), and Samet and Schmeidler (2001). ${ }^{16}$

Monotonicity requires that when the set of goods expands and the set of bads contracts for every agent, the choice should expand. Formally:

Monotonicity. For all $R, R^{\prime} \in \mathcal{D}$, if for all $i \in N, G\left(R_{i}\right) \subseteq G\left(R_{i}^{\prime}\right)$ and $B\left(R_{i}\right) \supseteq$ $B\left(R_{i}^{\prime}\right)$, then $\varphi(R) \subseteq \varphi\left(R^{\prime}\right)$.

The next axiom requires that the decision on each object should depend only on agents' evaluations of this object in terms of good, bad, or null. For all $R_{i} \in \mathcal{S}$ and all $x \in A$, let $\left.R_{i}\right|_{\{\{x\}, \varnothing\}}$ be the restriction of $R_{i}$ to $\{\{x\}, \varnothing\}$. Let $\left.R\right|_{\{\{x\}, \varnothing\}} \equiv\left(\left.R_{i}\right|_{\{\{x\}, \varnothing\}}\right)_{i \in N}$.

Independence. For all $x \in A$ and all $R, R^{\prime} \in \mathcal{D}$ with $\left.R\right|_{\{\{x\}, \varnothing\}}=\left.R^{\prime}\right|_{\{\{x\}, \varnothing\}}$,

$$
x \in \varphi(R) \Leftrightarrow x \in \varphi\left(R^{\prime}\right) .
$$

We consider the above axioms in conjunction with several combinations of the following standard axioms. We first define useful notation. Given each permutation on $N, \pi: N \rightarrow N$ and each $R \in \mathcal{D}$, let $\boldsymbol{R}^{\boldsymbol{\pi}} \equiv\left(R_{\pi(i)}\right)_{i \in N}$. For each $x, y \in A$ and each $X \in 2^{A}$, let $\boldsymbol{\rho}_{\boldsymbol{x}, \boldsymbol{y}}(\boldsymbol{X}) \in 2^{A}$ be such that $x \in \rho_{x, y}(X) \Leftrightarrow y \in X$, $y \in \rho_{x, y}(X) \Leftrightarrow x \in X$, and for all $z \in X \backslash\{x, y\}, z \in \rho_{x, y}(X)$. Thus, $\rho_{x, y}$ is the renaming operation that switch names between $x$ and $y$. Given $i \in N$, for each $R_{i} \in \mathcal{D}_{i}$, let $\boldsymbol{\rho}_{\boldsymbol{x}, \boldsymbol{y}} \boldsymbol{R}_{\boldsymbol{i}} \in \mathcal{D}_{i}$ be the preference where the roles of $x$ and $y$ in $R_{i}$ are switched: that is, for all $X, X^{\prime} \subseteq A, X \rho_{x, y} R_{i} X^{\prime} \Leftrightarrow \rho_{x, y}(X) R_{i} \rho_{x, y}\left(X^{\prime}\right)$. Then $x$ (or $y$ ) plays the same role in $R_{i}$ as $y$ (or $x$ ) does in $\rho_{x, y} R_{i}$. For each $R \in \mathcal{D}$, let $\boldsymbol{\rho}_{\boldsymbol{x}, \boldsymbol{y}} \boldsymbol{R} \equiv\left(\rho_{x, y} R_{i}\right)_{i \in N}$.

Voter sovereignty. For all $x \in A$, there exist $R, R^{\prime} \in \mathcal{D}$ such that $x \in \varphi(R)$ and $x \notin \varphi\left(R^{\prime}\right) .{ }^{17}$

Weak efficiency. For all $R \in \mathcal{D}$, there exists no $X \subseteq A$ such that for all $i \in N$, $X P_{i} \varphi(R)$.

$(\{a, c\},\{b\})$, and $\left(G\left(R_{3}\right), B\left(R_{3}\right)\right)=(\{a, b, c\}, \varnothing)$. Then $\varphi^{\text {pl-scoring }}(R)=\{a\}$. Let $R_{1}^{\prime}$ be such that $\left(G\left(R_{1}^{\prime}\right), B\left(R_{1}^{\prime}\right)\right)=(\{a\},\{b\})$. Then $\varphi^{\text {pl-scoring }}\left(R_{1}^{\prime}, R_{2}, R_{3}\right)=\{a, c\}$. Since $c$ is a null for both $R_{1}$ and $R_{1}^{\prime}$, this shows a violation of null-independence.

${ }^{16}$ Our axioms are stated differently from the axioms in these papers. We generalize their axioms in order to deal with non-linear preferences.

${ }^{17}$ Our voter sovereignty is weaker than "voter sovereignty" in Barberà, Sonnenschein, and Zhou (1991), which states that for all $X \in 2^{A}$, there exists $R \in \mathcal{D}$ such that $\varphi(R)=X$. 
Efficiency. For all $R \in \mathcal{D}$, there exists no $X \subseteq A$ such that for all $i \in N, X R_{i}$ $\varphi(R)$ and for some $j \in N, X P_{j} \varphi(R)$.

Anonymity. For all $R \in \mathcal{D}$ and all permutation $\pi$ on $N, \varphi(R)=\varphi\left(R^{\pi}\right)$.

Neutrality. For all $R \in \mathcal{D}$ and all $x, y \in A, x \in \varphi(R)$ if and only if $y \in \varphi\left(\rho_{x, y} R\right)$.

\section{Results}

\subsection{Characterizations of strategy-proof voting rules}

Throughout Section 4.1, we consider the separable domain (our results also apply to the additive domain) unless stated otherwise. We first characterize rules that satisfy monotonicity, independence, and some combinations of voter sovereignty, anonymity, and neutrality.

Proposition 1. A rule satisfies monotonicity and independence if and only if it is in Family $\Phi^{*}$.

See the proof in the Appendix.

Remark 1. In proving Proposition 1, we only use the three properties, A1-A3, of the separable domain. Thus, the same result also holds for any other subdomains satisfying these properties. ${ }^{18}$ Examples are subdomains in Example 1.

Within Family $\Phi^{*}$, it is easy to show that voter sovereignty, anonymity,

\footnotetext{
${ }^{18}$ For domains without properties A1-A3, Proposition 1 may not hold. Here is an example. For simplicity, let $A \equiv\{a, b, c\}$ and $N \equiv\{1,2\}$. Let $\mathcal{D}^{*}$ be the domain consisting of only the following three admissible preferences. First is a preference $R_{0}$, in which only $a$ is a good and both $b$ and $c$ are nulls. Second is a preference $R_{0}^{\prime}$, in which only $b$ is a good and both $a$ and $c$ are nulls. Last is a preference $R_{0}^{\prime \prime}$, in which $a$ is a good, $b$ is a bad, and $c$ is a null. Then clearly this domain $\mathcal{D}^{*}$ violates $\mathrm{A} 1$, since $a$ is never a bad and $c$ is always a null. Let $\varphi^{*}$ be the rule defined in the following table, where each cell represents the choice for the profile composed of its corresponding row and column preferences.
}

\begin{tabular}{|c|c|c|c|}
\hline & $R_{0}$ & $R_{0}^{\prime}$ & $R_{0}^{\prime \prime}$ \\
\hline$R_{0}$ & $\{a, b\}$ & $\{a, b\}$ & $\{a\}$ \\
\hline$R_{0}^{\prime}$ & $\{a, b\}$ & $\varnothing$ & $\{a\}$ \\
\hline$R_{0}^{\prime \prime}$ & $\{a\}$ & $\{a\}$ & $\{a\}$ \\
\hline
\end{tabular}

Note that the set of goods expands and the set of bads contracts only when preference changes from $R_{0}^{\prime \prime}$ to $R_{0}$. So clearly, $\varphi^{*}$ satisfies monotonicity. It is tedious to check independence of $\varphi^{*}$. However since $b \in \varphi^{*}\left(R_{0}, R_{0}\right)$ and $b \notin \varphi^{*}\left(R_{0}^{\prime}, R_{0}^{\prime}\right)$, then $\varphi^{*}$ violates objectwise monotonicity (see p.27 for the definition) and so is not a rule in Family $\Phi^{*}$. 
and neutrality are equivalent respectively to three properties, P-unanimity, Panonymity, and P-neutrality, of profiles of power structures. Therefore, we obtain the following corollary.

Corollary 1. (i) A rule satisfies monotonicity, independence, and voter sovereignty if and only if it is a rule in Family $\Phi^{*}$ associated with a P-unanimous profile of power structures.

(ii) A rule satisfies monotonicity, independence, and anonymity if and only if it is a rule in Family $\Phi^{*}$ associated with a P-anonymous profile of power structures. (iii) A rule satisfies monotonicity, independence, and neutrality if and only if it is a rule in Family $\Phi^{*}$ associated with a P-neutral profile of power structures.

The corresponding examples to show the independence of axioms in Proposition 1 and also in Corollary 1 can be easily obtained. Adding efficiency, we are left only with serially dictatorial rules in Family $\Phi^{*}$. Formally:

Proposition 2. A rule satisfies monotonicity, independence, and efficiency if and only if it is a serially dictatorial rule in Family $\Phi^{*}$.

Although we relax efficiency with weak efficiency, we cannot escape dictatorship.

Proposition 3. A rule satisfies monotonicity, independence, and weak efficiency if and only if it is a dictatorial rule in Family $\Phi^{*}$.

See the proofs of Propositions 2 and 3 in the Appendix A.

Remark 2. In proving Propositions 2 and 3, we use either one of the two properties, Property $\mathrm{P}$ and Property Q, of the separable domain. The two results can also be established for any subdomain satisfying Property P or Property Q. ${ }^{19}$ For domains not satisfying either one of the two properties, the results may not

${ }^{19}$ Properties A1-A3 are also required for Propositions 2 and 3. For example, in the domain $\mathcal{D}^{*}$, defined in the footnote of Remark 1 , let $\varphi$ be defined by the following table.

\begin{tabular}{|c|c|c|c|}
\hline$\varphi$ & $R_{0}$ & $R_{0}^{\prime}$ & $R_{0}^{\prime \prime}$ \\
\hline$R_{0}$ & $\{a, b\}$ & $\{a, b\}$ & $\{a\}$ \\
\hline$R_{0}^{\prime}$ & $\{a, b\}$ & $\{b\}$ & $\{a\}$ \\
\hline$R_{0}^{\prime \prime}$ & $\{a\}$ & $\{a\}$ & $\{a\}$ \\
\hline
\end{tabular}

Note that the set of goods expands and the set of bads contracts only when preference changes from $R_{0}^{\prime \prime}$ to $R_{0}$. So clearly, $\varphi$ satisfies monotonicity. It is tedious to show independence and efficiency of $\varphi$. Since agent 1 does not attain his best alternative at $\left(R_{0}^{\prime}, R_{0}^{\prime \prime}\right)$ and agent 2 does not attain his best alternative at $\left(R_{0}^{\prime \prime}, R_{0}^{\prime}\right)$, none of them can be a dictator. So $\varphi$ is not dictatorial. 
hold. For instance, when there are only two objects (see Example ?? for another example with more than three objects), there exist non-dictatorial rules satisfying the three axioms. For example, consider the following rule. Let $\tau:\left\{\left(C_{1}, C_{2}\right) \in \mathfrak{C}^{*}:\left|C_{1}\right|=\left|C_{2}\right|\right\} \rightarrow\{0,1\}$ be a function, called, tie-breaking function, such that for each pair $\left(C_{1}, C_{2}\right)$ of disjoint groups with the same size, either $\tau\left(C_{1}, C_{2}\right)=1$ or $\tau\left(C_{2}, C_{1}\right)=1$ but not both. Let $\varphi^{\tau}$ be the rule that makes the same decision on each object $x$ as the "plurality rule" whenever the group of agents in favor of $x$ and the group of agents against $x$ have different sizes. When the sizes of the two groups ties, the decision relies on the tie-breaking function $\tau$. Formally, for all $R \in \mathcal{S}^{N}$ and all $x \in X$, (i) if $\left|N_{x}^{G}(R)\right| \neq\left|N_{x}^{B}(R)\right|$, then $x \in \varphi^{\tau}(R, X) \Leftrightarrow\left|N_{x}^{G}(R)\right|>\left|N_{x}^{B}(R)\right|$; (ii) if $\left|N_{x}^{G}(R)\right|=\left|N_{x}^{B}(R)\right|$, then $x \in \varphi^{\tau}(R, X) \Leftrightarrow \tau\left(N_{x}^{G}(R), N_{x}^{B}(R)\right)=1$. By definition, $\varphi^{\tau}$, which is neither serially dictatorial nor dictatorial in Family $\Phi^{*}$, clearly satisfies monotonicity and independence. However, it is shown by $\mathrm{Ju}(2002 \mathrm{a})$ that $\varphi^{\tau}$ satisfies efficiency when there are only two objects. In the "variable agenda model", he characterizes the family of all such rules based on "non-manipulability", "restricted efficiency", and anonymity.

Example 4. Trichotomous or dichotomous preferences. Consider the trichotomous domain $\mathcal{S}_{\text {Tri }}^{N}$ and the dichotomous domain $\mathcal{S}_{\text {Di }}^{N}$, defined in Example 1. Every preference $R_{i}$ in both domains is represented by $U_{i}$ defined as follows: for all $X \subseteq A, U_{i}(X) \equiv\left|G\left(R_{i}\right) \cap X\right|-\left|B\left(R_{i}\right) \cap X\right|$. Note that for each profile $R \in \mathcal{S}_{\text {Tri }}^{N}$, the sum of utilities of $X, \sum_{i \in N} U_{i}(X)$, can be calculated in the following two equivalent ways: ${ }^{20}$

$$
\begin{aligned}
\sum_{i \in N} U_{i}(X) & =\sum_{i \in N}\left(\left|G\left(R_{i}\right) \cap X\right|-\left|B\left(R_{i}\right) \cap X\right|\right) \\
& =\sum_{x \in X}\left(\left|N_{x}^{G}(R)\right|-\left|N_{x}^{B}(R)\right|\right) .
\end{aligned}
$$

Clearly, $\mathcal{S}_{\text {Tri }}^{N}$ and $\mathcal{S}_{\mathrm{Di}}^{N}$ violate both Property $\mathrm{P}$ and $\mathrm{Q}$. We now show that over any one of these two domains, there are non-dictatorial voting rules, for example, plurality rule $\varphi^{\mathrm{pl}}$, satisfying the three requirements in Proposition $2 .{ }^{21}$ To explain this, let $R \in \mathcal{S}_{\text {Tri }}^{N}$ and $Y \equiv \varphi^{\mathrm{pl}}(R)$. Let $X \subseteq A$. We only have to show that if someone is better off in $X$ than in $Y$, then there is another who is worse off. Note first that for all $y \in Y,\left|N_{y}^{G}(R)\right|-\left|N_{y}^{B}(R)\right|>0$ and all $x \notin Y,\left|N_{x}^{G}(R)\right|-\left|N_{x}^{B}(R)\right| \leq 0$. Hence $\sum_{x \in X \backslash Y}\left(\left|N_{x}^{G}(R)\right|-\left|N_{x}^{B}(R)\right|\right) \leq 0$

\footnotetext{
${ }^{20}$ See Lemma 1 in Ju (2002c) for the proof.

${ }^{21} \mathrm{Ju}(2002 \mathrm{c})$ characterizes rules satisfying efficiency, independence, and anonymity over the two restricted domains, $\mathcal{S}_{\text {Tri }}^{N}$ and $\mathcal{S}_{\mathrm{Di}}^{N}$.
} 
and $\sum_{y \in Y \backslash X}\left(\left|N_{y}^{G}(R)\right|-\left|N_{y}^{B}(R)\right|\right)>0$ and so

$$
\begin{aligned}
& \sum_{x \in X \backslash Y}\left(\left|N_{x}^{G}(R)\right|-\left|N_{x}^{B}(R)\right|\right)+\sum_{x \in X \cap Y}\left(\left|N_{x}^{G}(R)\right|-\left|N_{x}^{B}(R)\right|\right) \\
\leq & \sum_{y \in Y \backslash X}\left(\left|N_{y}^{G}(R)\right|-\left|N_{y}^{B}(R)\right|\right)+\sum_{y \in X \cap Y}\left(\left|N_{y}^{G}(R)\right|-\left|N_{y}^{B}(R)\right|\right) .
\end{aligned}
$$

Therefore by $(*), \sum_{i \in N} U_{i}(X) \leq \sum_{i \in N} U_{i}(Y)$. It follows from this inequality that if someone is better off in $X$ than in $Y$, then there is another who is worse off.

We next establish the logical relation between the following two combinations of axioms; the combination of strategy-proofness and null-independence and the combination of monotonicity and independence. We show that within voting rules, the two combinations are equivalent.

Proposition 4. A voting rule satisfies strategy-proofness and null-independence if and only if it satisfies monotonicity and independence.

See the proof in the Appendix. Now we are ready to state our main results. It follows from Propositions 1 and 4 that rules in Family $\Phi^{*}$ are the only voting rules satisfying strategy-proofness and null-independence. Formally:

Theorem 1. A voting rule satisfies strategy-proofness and null-independence if and only if it is in Family $\Phi^{*}$.

Examples ??-?? establish the independence of strategy-proofness, null-independence, and votes-only property.

Example 5. Strategy-proofness and null-independence. Let $\varphi$ be such that for all $R \in \mathcal{D}, \varphi(R) \in \operatorname{Max}\left[R_{1}:\{\{x\}: x \in A\}\right]$. Then clearly, $\varphi$ satisfies strategyproofness and null-independence. However, $\varphi$ violates votes-only property.

Example 6. Strategy-proofness and votes-only property. Define $\varphi$ as follows. For each $R \in \mathcal{D}$, (i) if $G\left(R_{1}\right) \neq \varnothing, \varphi(R) \equiv G\left(R_{1}\right)$, (ii) if $G\left(R_{1}\right)=\varnothing$ and $B\left(R_{1}\right) \neq \varnothing$, $\varphi(R) \equiv A \backslash B\left(R_{1}\right)$, and (iii) if $G\left(R_{1}\right)=\varnothing=B\left(R_{1}\right), \varphi(R) \equiv \varnothing$. Clearly, $\varphi$ satisfies votes-only property. Since agent 1 always attains one of his best alternatives and $\varphi$ does not depend on any other's preference, $\varphi$ is strategy-proof. Suppose that $x$ is a null for both $R_{1}$ and $R_{1}^{\prime}$ and that part (ii) applies for $R_{1}$ and part (iii) applies for $R_{1}^{\prime}$. Then for all $R_{-1} \in \Pi_{N \backslash 1} \mathcal{D}_{i}, x \in \varphi\left(R_{1}, R_{-1}\right)$ and $x \notin \varphi\left(R_{1}^{\prime}, R_{-1}\right)$. Therefore $\varphi$ violates null-independence. 
Example 7. Null-independence and votes-only property. Consider the weighted plurality rule $\varphi^{\text {weight-pl }}$. Clearly, $\varphi^{\text {weight-pl }}$ satisfies null-independence and votes-only property. Let $x \in A$. Let $R \in \mathcal{D}$ be such that $\sum_{i \in N_{x}^{G}(R)} \frac{1}{\left|G\left(R_{i}\right)\right|}=\sum_{i \in N_{x}^{B}(R)} \frac{1}{\left|B\left(R_{i}\right)\right|}$ and for some $i \in N_{x}^{G}(R),\left|G\left(R_{i}\right)\right| \geq 2$ and if $X, X^{\prime} \subseteq A \backslash x, X \cup x P_{i} X^{\prime}$. Then $x \notin \varphi^{\text {weight-pl }}(R)$. However, if $i$ reports $R_{i}^{\prime}$ such that $G\left(R_{i}^{\prime}\right)=\{x\}$, then $x \in \varphi^{\text {weight-pl }}\left(R_{i}^{\prime}, R_{-i}\right)$ and so $\varphi^{\text {weight-pl }}\left(R_{i}^{\prime}, R_{-i}\right) \quad P_{i} \varphi^{\text {weight-pl }}\left(R_{i}, R_{-i}\right)$, violating strategy-proofness.

It follows from Proposition 4 and Corollary 1 that:

Corollary 2. (i) A voting rule satisfies strategy-proofness, null-independence, and voter sovereignty if and only if it is a rule in Family $\Phi^{*}$, associated with a P-unanimous profile of power structures.

(ii) A voting rule satisfies strategy-proofness, null-independence, and anonymity if and only if it is a rule in Family $\Phi^{*}$, associated with a P-anonymous profile of power structures.

(iii) A voting rule satisfies strategy-proofness, null-independence, and neutrality if and only if it is a rule in Family $\Phi^{*}$, associated with a P-neutral profile of power structures.

Remark 3. In proving Proposition 4, Theorem 1, and Corollary 2, Property R plays a critical role. Therefore, the same results can be established for any subdomain satisfying this property. These results may not hold for domains not satisfying Property R. See Example ?? below.

Example 8. A strategy-proof and null-independent rule over the dichotomous domain $\mathcal{S}_{D i}^{N}$, which is not in Family $\Phi^{*}$. Define $\varphi^{*}$ as follows. Fix two objects $a^{*}$ and $b^{*}$. For all $R \in \mathcal{S}_{\mathrm{Di}}^{N}$ and all $x \in A \backslash a^{*}, x \in \varphi^{*}(R) \Leftrightarrow\left|N_{x}^{G}(R)\right|>$ $\left|N_{x}^{B}(R)\right|$; if $\left|N_{a^{*}}^{G}(R)\right| \neq\left|N_{a^{*}}^{B}(R)\right|$, then $a^{*} \in \varphi^{*}(R) \Leftrightarrow\left|N_{a^{*}}^{G}(R)\right|>\left|N_{a^{*}}^{B}(R)\right|$ and if $\left|N_{a^{*}}^{G}(R)\right|=\left|N_{a^{*}}^{B}(R)\right|$, then $a^{*} \in \varphi^{*}(R) \Leftrightarrow\left[b^{*} \in \varphi^{*}(R)\right]$.

Note that when the number of agents is even, $\varphi^{*}$ clearly is not in Family $\Phi^{*}$. Note also that for all $R \in \mathcal{S}_{\mathrm{Di}}^{N}, \varphi^{*}(R) \backslash a^{*}=\varphi^{\mathrm{pl}}(R) \backslash a^{*}$ and that when $a^{*} \in \varphi^{\mathrm{pl}}(R)$, $\varphi^{*}(R)=\varphi^{\mathrm{pl}}(R)$ and when $a^{*} \notin \varphi^{\mathrm{pl}}(R), \varphi^{*}(R)=\varphi^{\mathrm{pl}}(R) \cup a^{*}$ if and only if $a^{*}$ makes a tie at $R$ and $b^{*} \in \varphi^{\mathrm{pl}}(R)$. Decisions by $\varphi^{*}$ do not depend on agents' labels (or names). So $\varphi^{*}$ is anonymous. Since $\mathcal{S}_{\mathrm{Di}}^{N}$ does not allow for nulls, $\varphi^{*}$ satisfies null-independence vacuously. To give an intuition for strategy-proofness, consider $R \in \mathcal{S}_{\mathrm{Di}}^{N}$ at which both $a^{*}$ and $b^{*}$ make ties, that is, $\left|N_{a^{*}}^{G}(R)\right|=\left|N_{a^{*}}^{B}(R)\right|$ and $\left|N_{b^{*}}^{G}(R)\right|=\left|N_{b^{*}}^{B}(R)\right|$. Then by definition, $b^{*}$ is rejected and so $a^{*}$ is also rejected at $R$. Consider an agent $i \in N$ for whom $a^{*}$ is a good and $b^{*}$ is a bad. He can make $a^{*}$ accepted only by reporting $b^{*}$ as a good and such a misrepresentation will make 
$b^{*}$, which is a bad for him, accepted also. Since his preference is dichotomous, the utility from $a^{*}$ will be cancelled out by the disutility from $b^{*}$. Therefore, such a misrepresentation will not benefit him. Considering different cases, one by one, we can show that no one can ever be benefited by misrepresenting his preference. $^{22}$

Over the linear separable domain $\mathcal{S}_{\text {lin }}^{N}$, null-independence is vacuously satisfied by any rule. Thus, it follows from Corollary 2 and Remark 3 that:

Corollary 3. Over the linear separable domain $\mathcal{S}_{\text {lin }}^{N}$, schemes of voting by committees are the only voting rules satisfying strategy-proofness and voter sovereignty.

By Propositions 2, 3, and 4, we characterize serially dictatorial or dictatorial rules in Family $\Phi^{*}$. Formally:

Theorem 2. (i) A voting rule satisfies strategy-proofness, null-independence, and efficiency if and only if it is a serially dictatorial rule in Family $\Phi^{*}$.

(ii) A voting rule satisfies strategy-proofness, null-independence, and weak efficiency if and only if it is a dictatorial rule in Family $\Phi^{*} \cdot{ }^{23}$

Remark 4. This result can also be obtained over any subdomain satisfying Property $\mathrm{R}$ and either Property $\mathrm{P}$ or Property Q. If the domain violates these properties, the result may not hold. For example, when there are only two objects, neither Property $\mathrm{P}$ nor Property $\mathrm{Q}$ holds and the non-dictatorial rule defined in Remark ?? satisfies strategy-proofness, null-independence, and efficiency. Another examples are the trichotomous domain and the dichotomous domain. As explained in Example ??, the plurality rule satisfies all the three axioms.

\subsection{The maximal domain result}

In this section, we study how further the domain can be enlarged in order for rules in Family $\Phi^{*}$ to be strategy-proof. To address this question, we need a more general definition of rules in Family $\Phi^{*}$. Our previous definition is made in terms of the set of goods and the set of bads, which are well-defined only for separable preferences. Note that the set of goods coincides with the intersection of all "top (or best) alternatives" and the set of bads coincides with the intersection of all "bottom (or worst) alternatives". We use these intersections to extend the definition of rules in Family $\Phi^{*}$.

\footnotetext{
${ }^{22}$ Formal proof is provided in the Appendix of $\mathrm{Ju}$ (2002c). He also shows that over the dichotomous domain, $\varphi^{*}$ satisfies efficiency.

${ }^{23}$ Examples showing independence of axioms in Theorem 2 are available upon request.
} 
For all preferences $R_{i}$, let $\boldsymbol{T}^{*}\left(\boldsymbol{R}_{\boldsymbol{i}}\right)$ be the intersection of all top alternatives and $\boldsymbol{B}^{*}\left(\boldsymbol{R}_{\boldsymbol{i}}\right)$ the intersection of all bottom alternatives. For example, when $R_{i}$ is separable, $T^{*}\left(R_{i}\right)=G\left(R_{i}\right)$ and $B^{*}\left(R_{i}\right)=B\left(R_{i}\right)$. For each preference profile $R$, let $\boldsymbol{N}_{\boldsymbol{x}}^{\boldsymbol{T}^{*}}(\boldsymbol{R}) \equiv\left\{i \in N: x \in T^{*}\left(R_{i}\right)\right\}$ and $\boldsymbol{N}_{\boldsymbol{x}}^{\boldsymbol{B}^{*}}(\boldsymbol{R}) \equiv\left\{i \in N: x \in B^{*}\left(R_{i}\right)\right\}$. We extend the definition of Family $\Phi^{*}$, replacing $N_{x}^{G}(R)$ and $N_{x}^{B}(R)$ in the previous definition with $N_{x}^{T^{*}}(R)$ and $N_{x}^{B^{*}}(R)$ respectively. More precisely, a rule $\varphi$ is in Family $\Phi^{*}$ if for each object $x \in A$, there exists a power structure $\mathfrak{C}_{x}$ such that for all $R \in \mathcal{D}, x \in \varphi(R) \Leftrightarrow\left(N_{x}^{T^{*}}(R), N_{x}^{B^{*}}(R)\right) \in \mathfrak{C}_{x}$.

Throughout this section, we restrict our attention to domains in which any object that is either in all top alternatives or in all bottom alternatives is critical when added to any alternative not including it. The addition either makes an improvement or the opposite. Formally:

Assumption D. For all $i \in N$, all $R_{i} \in \mathcal{D}_{i}$, all $x \in T^{*}\left(R_{i}\right) \cup B^{*}\left(R_{i}\right)$, and all $X \subseteq A \backslash x$, either $X \cup x P_{i} X$ or $X P_{i} X \cup x$.

Clearly, both the separable domain and the additive domain satisfy this assumption. However, it has nothing to do with the separability restriction. Indeed, any domain consisting of linear preferences satisfies Assumption D.

We further focus on domains that have enough variety of preferences in the following sense.

Definition. Domain $\mathcal{D}$ is rich if for all $i \in N$ and all disjoint alternatives $X, X^{\prime} \subseteq A$, there exists $R_{i} \in \mathcal{D}_{i}$ such that $T^{*}\left(R_{i}\right)=X$ and $B^{*}\left(R_{i}\right)=X^{\prime}$.

Note that the richness property is an extension of property A4, which applies to subdomains of the separable domain. It corresponds to the richness property considered by Barberà, Sonnenschein, and Zhou (1991, p.605).

Given a power structure $\mathfrak{C}$, a pair $\left(C_{1}, C_{2}\right) \in \mathfrak{C}$ is minimal in $\mathfrak{C}$ if there exists no $\left(C_{1}^{\prime}, C_{2}^{\prime}\right) \in \mathfrak{C} \backslash\left(C_{1}, C_{2}\right)$ such that $C_{1}^{\prime} \subseteq C_{1}$ and $C_{2}^{\prime} \supseteq C_{2}$. A pair $\left(C_{1}, C_{2}\right) \in \mathfrak{C}^{*} \backslash \mathfrak{C}$ is maximal in $\mathfrak{C}^{*} \backslash \mathfrak{C}$ if there exists no $\left(C_{1}^{\prime}, C_{2}^{\prime}\right) \in \mathfrak{C}^{*} \backslash \mathfrak{C}$ such that $\left(C_{1}^{\prime}, C_{2}^{\prime}\right) \neq$ $\left(C_{1}, C_{2}\right), C_{1}^{\prime} \supseteq C_{1}$, and $C_{2}^{\prime} \subseteq C_{2}$. Let $\varphi$ be a rule in Family $\Phi^{*}$ associated with profile $\left(\mathfrak{C}_{x}\right)_{x \in A}$. Agent $i \in N$ is a dummy of object $\boldsymbol{x}$ if either (i) for all minimal pair $\left(C_{1}, C_{2}\right)$ in $\mathfrak{C}_{x}, i \notin C_{1}$ or (ii) for all maximal pair $\left(C_{1}, C_{2}\right)$ in $\mathfrak{C}^{*} \backslash \mathfrak{C}_{x}, i \notin C_{2} .{ }^{24}$ Thus, the dummy agent does not play any essential role in accepting or rejecting $x$. Agent $i \in N$ is a vetoer of object $\boldsymbol{x}$ if either for all $\left(C_{1}, C_{2}\right) \in \mathfrak{C}_{x}, i \notin C_{2}$ or for all $\left(C_{1}, C_{2}\right) \in \mathfrak{C}^{*} \backslash \mathfrak{C}_{x}, i \notin C_{1}{ }^{25}$ Thus a vetoer always has an option to reject or

\footnotetext{
${ }^{24}$ The definition of dummy is a natural extension of the corresponding notion in Barberà, Sonnenschein, and Zhou (1991, p.606). In the linear domain, they coincide with each other.

${ }^{25}$ The definition of vetoer is a natural extension of the corresponding notion in Barberà,
} 
accept $x$. We are interested in rules in Family $\Phi^{*}$ that do not have any dummy or vetoer.

Fact 1. A preference $R_{i}$ is separable if and only if for all $x \in A$ and all $X \subseteq A \backslash x$,

$$
\begin{aligned}
& {[X \cup x] P_{i} X \quad \Leftrightarrow \quad x \in T^{*}\left(R_{i}\right)} \\
& X P_{i}[X \cup x] \Leftrightarrow x \in B^{*}\left(R_{i}\right) .
\end{aligned}
$$

Proof. When $R_{i}$ is separable, $T^{*}\left(R_{i}\right)=G\left(R_{i}\right)$ and $B^{*}\left(R_{i}\right)=B\left(R_{i}\right)$ and $(1)$ and (2) hold. To prove the converse, suppose (1) and (2). Then for all $x \in A$ and all $X \subseteq A \backslash x$

$$
\begin{aligned}
& {[X \cup x] P_{i} X \quad \Leftrightarrow \quad x P_{i} \varnothing ;} \\
& X P_{i}[X \cup x] \Leftrightarrow \varnothing P_{i} x,
\end{aligned}
$$

which is evidently equivalent to separability.

Proposition 5. For all rules in Family $\Phi^{*}$ without any dummy or vetoer, if the rule is strategy-proof over a rich domain, then the domain is a subdomain of the separable domain.

Proof. Let $\mathcal{D}$ be a rich domain. Let $\varphi: \mathcal{D} \rightarrow 2^{A}$ be a rule in Family $\Phi^{*}$, associated with $\left(\mathfrak{C}_{x}\right)_{x \in A}$. Suppose that $\varphi$ does not have any vetoer or dummy and that $\varphi$ is strategy-proof. Let $i \in N$ and $R_{i} \in \mathcal{D}_{i}$. Let $x \in A$ and $Y \subseteq A \backslash x$. Note that since agent $i$ is not a dummy, there exist minimal pair $\left(C_{1}, C_{2}\right)$ in $\mathfrak{C}_{x}$ with $i \in C_{1}$ and maximal pair $\left(C_{1}^{0}, C_{2}^{0}\right)$ in $\mathfrak{C}^{*} \backslash \mathfrak{C}_{x}$ with $i \in C_{2}^{0}$. Also since $i$ is not a vetoer, for all $y \in A,(N \backslash i, i) \in \mathfrak{C}_{y}$ and $(i, N \backslash i) \notin \mathfrak{C}_{y} \cdot{ }^{26}$

We first show (1) in Fact 1 . Suppose $x \in T^{*}\left(R_{i}\right)$. For each $j \neq i$, let $R_{j}$ be such that

$$
\begin{aligned}
& T^{*}\left(R_{j}\right)=Y \cup x \text { and } B^{*}\left(R_{j}\right)=A \backslash(Y \cup x), \text { if } j \in C_{1} ; \\
& T^{*}\left(R_{j}\right)=Y \text { and } B^{*}\left(R_{j}\right)=A \backslash Y, \text { if } j \in C_{2} ; \\
& T^{*}\left(R_{j}\right)=Y \text { and } B^{*}\left(R_{j}\right)=A \backslash(Y \cup x) \text {, if } j \notin C_{1} \cup C_{2} .
\end{aligned}
$$

Note that $\left(N_{x}^{T^{*}}(R), N_{x}^{B^{*}}(R)\right)=\left(C_{1}, C_{2}\right)$; for all $y \in Y, N_{y}^{T^{*}}(R) \supseteq N \backslash i$ and $N_{y}^{B^{*}}(R) \subseteq i$; for all $y \in A \backslash(Y \cup x), N_{y}^{T^{*}}(R) \subseteq i$ and $N_{y}^{B^{*}}(R) \supseteq N \backslash i$. Since $(N \backslash i, i) \in \mathfrak{C}_{y}$ for all $y \in A$, then $Y \subseteq \varphi(R)$. Since $(i, N \backslash i) \notin \mathfrak{C}_{y}$ for all $y \in A$, then $\varphi(R) \cap(A \backslash(Y \cup x))=\varnothing$. Hence $\varphi(R)=Y \cup x$ or $Y$. Since $\left(C_{1}, C_{2}\right) \in \mathfrak{C}_{x}$,

Sonnenschein, and Zhou (1991, p.606). In the linear domain, the two notions coincide with each other.

${ }^{26}$ Since there is no vetoer, then for all $y \in A$, both $\mathfrak{C}_{y}$ and $\mathfrak{C}^{*} \backslash \mathfrak{C}_{y}$ are nonempty. So by P-monotonicity, $(N \backslash i, i) \in \mathfrak{C}_{y}$ and $(i, N \backslash i) \notin \mathfrak{C}_{y}$, for all $i \in N$. 
$\varphi(R)=Y \cup x$. Now let $R_{i}^{\prime}$ be such that $x \notin T^{*}\left(R_{i}^{\prime}\right)$. Then $N_{x}^{T^{*}}\left(R_{i}^{\prime}, R_{-i}\right)=C_{1} \backslash i$ and $N_{x}^{B^{*}}\left(R_{i}^{\prime}, R_{-i}\right) \supseteq N_{x}^{B^{*}}(R)=C_{2}$. Since $\left(C_{1}, C_{2}\right)$ is minimal, $x \notin \varphi\left(R_{i}^{\prime}, R_{-i}\right)$ and so $\varphi\left(R_{i}^{\prime}, R_{-i}\right)=Y$. By strategy-proofness, $[Y \cup x] R_{i} Y$. By Assumption D, $[Y \cup x] P_{i} Y$.

To prove the converse of (1), suppose $x \notin T^{*}\left(R_{i}\right)$. Then $N_{x}^{T^{*}}(R)=C_{1} \backslash i$ and $N_{x}^{B^{*}}(R)=C_{2}$ or $C_{2} \cup i$. Since $\left(C_{1}, C_{2}\right)$ minimal, $x \notin \varphi(R)$ and $\varphi(R)=Y$. Now let $R_{i}^{\prime \prime}$ be such that $x \in T^{*}\left(R_{i}^{\prime \prime}\right)$. Then $\varphi\left(R_{i}^{\prime \prime}, R_{-i}\right)=Y \cup x$. Therefore, by strategy-proofness, $Y R_{i}[Y \cup x]$.

Next we show (2) in Fact 1 . Suppose $x \in B^{*}\left(R_{i}\right)$. For each $j \neq i$, let $R_{j}$ be such that

$$
\begin{aligned}
& T^{*}\left(R_{j}\right)=Y \cup x \text { and } B^{*}\left(R_{j}\right)=A \backslash(Y \cup x), \text { if } j \in C_{1}^{0} ; \\
& T^{*}\left(R_{j}\right)=Y \text { and } B^{*}\left(R_{j}\right)=A \backslash Y, \text { if } j \in C_{2}^{0} ; \\
& T^{*}\left(R_{j}\right)=Y \text { and } B^{*}\left(R_{j}\right)=A \backslash(Y \cup x) \text {, if } j \notin C_{1}^{0} \cup C_{2}^{0} .
\end{aligned}
$$

Note that $\left(N_{x}^{T^{*}}(R), N_{x}^{B^{*}}(R)\right)=\left(C_{1}^{0}, C_{2}^{0}\right)$; for all $y \in Y, N_{y}^{T^{*}}(R) \supseteq N \backslash i$ and $N_{y}^{B^{*}}(R) \subseteq i$; for all $y \in A \backslash(Y \cup x), N_{y}^{T^{*}}(R) \subseteq i$ and $N_{y}^{B^{*}}(R) \supseteq N \backslash i$. Then for the same reason as above, $\varphi(R)=Y \cup x$ or $Y$. Since $\left(C_{1}^{0}, C_{2}^{0}\right) \notin \mathfrak{C}_{x}, \varphi(R)=Y$. Now let $R_{i}^{\#}$ be such that $x \notin B^{*}\left(R_{i}^{\#}\right)$. Then $N_{x}^{T^{*}}\left(R_{i}^{\#}, R_{-i}\right) \supseteq C_{1}^{0}$ and $N_{x}^{B^{*}}\left(R_{i}^{\#}, R_{-i}\right)=$ $C_{2}^{0} \backslash i$. Since $\left(C_{1}^{0}, C_{2}^{0}\right)$ is maximal in $\mathfrak{C}^{*} \backslash \mathfrak{C}_{x}, x \in \varphi\left(R_{i}^{\#}, R_{-i}\right)$. Hence $\varphi\left(R_{i}^{\#}, R_{-i}\right)=$ $Y \cup x$. By strategy-proofness, $Y R_{i}[Y \cup x]$. By Assumption D, $Y P_{i}[Y \cup x]$.

To prove the converse of $(2)$, suppose $x \notin B^{*}\left(R_{i}\right)$. Then (i) $N_{x}^{T^{*}}(R)=C_{1}$ or $C_{1} \cup i$ and (ii) $N_{x}^{B^{*}}(R)=C_{2} \backslash i$. Since $\left(C_{1}, C_{2}\right)$ maximal, $x \in \varphi(R)$ and $\varphi(R)=$ $Y \cup x$. Now let $R_{i}^{\# \#}$ be such that $x \in B^{*}\left(R_{i}^{\# \#}\right)$. Then $\varphi\left(R_{i}^{\# \#}, R_{-i}\right)=Y$. Therefore, by strategy-proofness, $[Y \cup x] R_{i} Y$.

It follows from Theorem 1 and Proposition 5 that:

Theorem 3. The separable domain is the unique maximal domain, within rich domains, over which every rule in Family $\Phi^{*}$, without any vetoer or dummy, is strategy-proof.

\section{Concluding remarks}

Our impossibility result, Theorem 2, relies on domain properties, Property $\mathrm{P}$ or Property Q. There are various restricted subdomains that do not satisfy these properties. Examples are the trichotomous domain $\mathcal{S}_{\text {Tri }}^{N}$ and the dichotomous domain $\mathcal{S}_{\mathrm{Di}}^{N}$ defined in Example ??. Characterizations of strategy-proof and efficient rules over these subdomains are established by Ju (2002c). 
Voting rules can be efficient at least over some subdomains, although not over the entire separable domain. Since there are finite numbers of objects and agents, every voting rule have possibly multiple maximal subdomains over which it is efficient. It would be interesting to identify these maximal domains, particularly for some standard rules such as "plurality rule" and "unanimity rule".

Over the linear separable domain, it is shown by Barberà, Sonnenschein, and Zhou (1991) that strategy-proofness and a slightly stronger version of voter sovereignty imply "tops-only" property, which corresponds to votes-only property in our preference domain. However, when non-linear preferences are admissible also, this implication no longer holds. The following example shows this.

Example 9. Let $\varphi$ be defined as follows. Fix $a, b \in A$. For all $R \in \mathcal{D}$, if $a P_{1} b$, then $\varphi(R) \equiv A \backslash B\left(R_{1}\right)$; otherwise, $\varphi(R) \equiv G\left(R_{1}\right)$. It is easy to show that $\varphi$ satisfies strategy-proofness and the notion of "voter sovereignty" in Barberà, Sonnenschein, and Zhou (1991). Clearly, $\varphi$ violates votes-only property since it relies on the ordering between $a$ and $b$.

The above rule is dictatorial. However, using the similar idea in its definition, we can also define non-dictatorial rules violating votes-only property.

Strategy-proofness pertains to the strategic misrepresentation of preferences by a single agent. When agents can form coalitions and manipulate the outcome jointly misrepresenting their preferences, we need a stronger requirement to prevent such manipulation.

Coalitional strategy-proofness. For all $R \in \mathcal{S}^{N}$, all $N^{\prime} \subseteq N$, and all $R_{N^{\prime}}^{\prime} \in$ $\mathcal{S}^{N^{\prime}}$, if $\varphi\left(R_{N^{\prime}}^{\prime}, R_{-N^{\prime}}\right) P_{i} \varphi(R)$ for some $i \in N^{\prime}$, then $\varphi(R) P_{j} \varphi\left(R_{N^{\prime}}^{\prime}, R_{-N^{\prime}}\right)$, for some $j \in N^{\prime}$.

We refer readers to Moulin (1993) for a survey of literature on coalitional strategy-proofness. When a rule in Family $\Phi^{*}$ satisfies this requirement and the full-range condition, coalitional strategy-proofness, applied to the grand coalition $N$, implies efficiency. Thus, by Theorem 2, this rule is serially dictatorial. However, serial dictatorship violates coalitional strategy-proofness. To prove this, consider the serially dictatorial rule associated with permutation $\pi$. Let $R$ be the profile in which all objects other than $a$ are null for all agents, $a$ is a null for agent $\pi(1), a$ is a good for agent $\pi(2)$, and $a$ is a bad for $\pi(3)$. Then $a$ will be chosen for $R$. But if $\pi(1)$ and $\pi(3)$ make a coalition and report jointly $\left(R_{1}^{\prime}, R_{3}\right)$ such that all objects except $a$ are nulls for $R_{1}^{\prime}$ and $a$ is a bad for $R_{1}^{\prime}$, then $a$ will not be chosen making $\pi$ (3) better off, without making $\pi(1)$ worse off. Therefore, there is no coalitionally strategy-proof rule satisfying the full-range condition within 
Family $\Phi^{*}$. Since any rule in Family $\Phi^{*}$, satisfying voter sovereignty, satisfies the full-range condition, we have the following result.

Corollary 4. If there are at least three agents, then there exists no voting rule that satisfies coalitional strategy-proofness, null-independence, and voter sovereignty.

When there are only two agents, serially dictatorial rules in Family $\Phi^{*}$ satisfy all the three requirements. In the two agents case, since the grand coalition is the only non-singleton coalition, it can be easily shown that the combination of coalitional strategy-proofness and voter sovereignty is equivalent to the combination of strategy-proofness and efficiency. When there are at least three agents, dropping voter sovereignty, we are left with only a small subfamily of Family $\Phi^{*}$, containing only the rules that either always select or never select each object, possibly except, at most, one object (see Ju, 2002b, for more details).

\section{A Proofs}

Throughout this section, let $\mathcal{D} \in\left\{\mathcal{S}^{N}, \mathcal{S}_{\text {add }}^{N}\right\}$.

To prove Proposition 1, we show that the combination of monotonicity and independence is equivalent to the following property. A rule $\varphi$ is objectwise monotonic if for all $x \in A$ and all $R, R^{\prime} \in \mathcal{D}$ with $N_{x}^{G}(R) \subseteq N_{x}^{G}\left(R^{\prime}\right)$ and $N_{x}^{B}(R) \supseteq N_{x}^{B}\left(R^{\prime}\right), x \in \varphi(R) \Rightarrow x \in \varphi\left(R^{\prime}\right) .{ }^{27}$ We need the following lemma.

Lemma 1. For all $R, R^{\prime} \in \mathcal{D}$ and all $x \in A$, if $N_{x}^{G}(R) \subseteq N_{x}^{G}\left(R^{\prime}\right)$ and $N_{x}^{B}(R) \supseteq$ $N_{x}^{B}\left(R^{\prime}\right)$, then there exists $\bar{R} \in \mathcal{D}$ such that $\left.\left.\bar{R}\right|_{\{\{x\}, \varnothing\}} \equiv R^{\prime}\right|_{\{\{x\}, \varnothing\}}$ and for all $i \in N$, $G\left(\bar{R}_{i}\right) \supseteq G\left(R_{i}\right)$ and $B\left(\bar{R}_{i}\right) \subseteq B\left(R_{i}\right)$.

Proof. Let $R, R^{\prime} \in \mathcal{D}$ and $x \in A$ be such that $N_{x}^{G}(R) \subseteq N_{x}^{G}\left(R^{\prime}\right), N_{x}^{B}(R) \supseteq$ $N_{x}^{B}\left(R^{\prime}\right)$. Since $N_{x}^{G}(R) \subseteq N_{x}^{G}\left(R^{\prime}\right)$ and $N_{x}^{B}(R) \supseteq N_{x}^{B}\left(R^{\prime}\right)$, then $N$ is partitioned into the following five subsets, $N_{x}^{G}(R), N_{x}^{G}\left(R^{\prime}\right) \backslash N_{x}^{G}(R), N_{x}^{B}\left(R^{\prime}\right), N_{x}^{B}(R) \backslash\left(N_{x}^{B}\left(R^{\prime}\right) \cup\right.$ $\left.N_{x}^{G}\left(R^{\prime}\right)\right)$, and $N \backslash\left(N_{x}^{G}\left(R^{\prime}\right) \cup N_{x}^{B}(R)\right)$.

For all $i \in N_{x}^{G}(R) \cup N_{x}^{B}\left(R^{\prime}\right) \cup\left[N \backslash\left(N_{x}^{G}\left(R^{\prime}\right) \cup N_{x}^{B}(R)\right)\right]$, let $\bar{R}_{i}=R_{i}$. By A2, for all $i \in N_{x}^{G}\left(R^{\prime}\right) \backslash N_{x}^{G}(R)$, there exists $\bar{R}_{i} \in \mathcal{D}_{i}$ such that $G\left(\bar{R}_{i}\right) \supseteq G\left(R_{i}\right) \cup\{x\}$ and $B\left(\bar{R}_{i}\right) \subseteq B\left(R_{i}\right) \backslash\{x\}$. By A3, for all $i \in N_{x}^{B}(R) \backslash\left(N_{x}^{B}\left(R^{\prime}\right) \cup N_{x}^{G}\left(R^{\prime}\right)\right)$, there exists $\bar{R}_{i} \in \mathcal{D}_{i}$ such that $G\left(\bar{R}_{i}\right) \supseteq G\left(R_{i}\right), B\left(\bar{R}_{i}\right) \subseteq B\left(R_{i}\right)$, and $x \notin G\left(\bar{R}_{i}\right) \cup B\left(\bar{R}_{i}\right)$.

\footnotetext{
${ }^{27}$ Objectwise monotonicity is similar to "monotonicity" or "non-negative responsiveness" in the classical voting model. See the "monotonicity" in Murakami (1966), the condition of "positive association" in Arrow (1964), and the "monotonicity" in Blau (1957). See also Aizerman and Aleskerov (1986) and Aleskerov and Duggan (1993).
} 
Then clearly, for all $i \in N, G\left(\bar{R}_{i}\right) \supseteq G\left(R_{i}\right), B\left(\bar{R}_{i}\right) \subseteq B\left(R_{i}\right)$, and $\left.\bar{R}_{i}\right|_{\{\{x\}, \varnothing\}} \equiv$

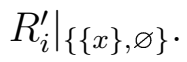

Lemma 2. A rule satisfies monotonicity and independence if and only if it satisfies objectwise monotonicity.

Proof. We only prove that both monotonicity and independence imply objectwise monotonicity. We omit the remaining part.

Let $\varphi: \mathcal{D} \rightarrow 2^{A}$ satisfy monotonicity and independence. Let $x \in A$ and $R, R^{\prime} \in \mathcal{D}$ be such that $N_{x}^{G}(R) \subseteq N_{x}^{G}\left(R^{\prime}\right), N_{x}^{B}(R) \supseteq N_{x}^{B}\left(R^{\prime}\right)$, and $x \in \varphi(R)$. By Lemma 1, there exists $\bar{R}$ such that $\left.\left.\bar{R}\right|_{\{\{x\}, \varnothing\}} \equiv R^{\prime}\right|_{\{\{x\}, \varnothing\}}$ and for all $i \in N$, $G\left(\bar{R}_{i}\right) \supseteq G\left(R_{i}\right)$ and $B\left(\bar{R}_{i}\right) \subseteq B\left(R_{i}\right)$. Therefore by monotonicity, $x \in \varphi(R)$ implies $x \in \varphi(\bar{R})$. Since $\left.\bar{R}_{i}\right|_{\{\{x\}, \varnothing\}}=\left.R_{i}^{\prime}\right|_{\{\{x\}, \varnothing\}}$, then by independence, $x \in \varphi\left(R^{\prime}\right)$.

We next show that rules in Family $\Phi^{*}$ are the only rules satisfying monotonicity and independence.

Proof of Proposition 1. Clearly, every rule in Family $\Phi^{*}$ satisfies monotonicity and independence. Let $\varphi$ satisfy the monotonicity and independence. Then by Lemma $2, \varphi$ also satisfies objectwise monotonicity.

Let $x \in A$. Let $\mathfrak{C}_{x}$ be defined as follows: $\left(C_{1}, C_{2}\right) \in \mathfrak{C}_{x}$ if and only if $C_{1} \cap C_{2}=$ $\varnothing$ and there exists $R \in \mathcal{D}$ such that $N_{x}^{G}(R) \subseteq C_{1}, N_{x}^{B}(R) \supseteq C_{2}$, and $x \in \varphi(R)$. Then by objectwise monotonicity, for each $x \in A, \mathfrak{C}_{x}$ satisfies P-monotonicity. Let $\hat{\varphi}$ be the rule in Family $\Phi^{*}$, associated with $\left(\mathfrak{C}_{x}\right)_{x \in A}$. We only have to show that $\varphi=\hat{\varphi}$. Let $R \in \mathcal{D}$. If $x \in \varphi(R)$, then $\left(N_{x}^{G}(R), N_{x}^{B}(R)\right) \in \mathfrak{C}_{x}$. Therefore $x \in \hat{\varphi}(R)$. Hence $\varphi(R) \subseteq \hat{\varphi}(R)$. Let $x \in \hat{\varphi}(R)$. Then $\left(N_{x}^{G}(R), N_{x}^{B}(R)\right) \in \mathfrak{C}_{x}$. Therefore there exists $R^{\prime} \in \mathcal{D}$ such that $N_{x}^{G}\left(R^{\prime}\right) \subseteq N_{x}^{G}(R), N_{x}^{B}\left(R^{\prime}\right) \supseteq N_{x}^{B}(R)$, and $x \in \varphi\left(R^{\prime}\right)$. By objectwise monotonicity, $x \in \varphi(R)$. Hence $\varphi(R) \supseteq \hat{\varphi}(R)$.

In order to prove Propositions 2 and 3, we use a sequence of lemmas similar to those in the proof of Arrow's Impossibility Theorem. ${ }^{28}$ Thus the following notions of "decisiveness" are needed. Let $\varphi$ be a rule. Let $M \subseteq N$ and $x \in A$. A group $S \subseteq N \backslash M$ is positively $M$-posterior-decisive for $x$, or simply, positively $\boldsymbol{M}$-decisive for $\boldsymbol{x}$ if for all $R \in \mathcal{D}$ with $M \subseteq N \backslash\left(N_{x}^{G}(R) \cup N_{x}^{B}(R)\right)$,

$$
S \subseteq N_{x}^{G}(R) \Rightarrow x \in \varphi(R) .
$$

When $M=\varnothing$, we say $S$ is positively decisive for $\boldsymbol{x}$. A group $S \subseteq N \backslash M$ is positively $M$-decisive if the group is positively $L$-decisive for all objects.

\footnotetext{
${ }^{28}$ Arrow (1964).
} 
When $M=\varnothing$, we say $S$ is positively decisive. A group $S \subseteq N \backslash M$ is negatively $M$-decisive for $\boldsymbol{x}$ if for all $R \in \mathcal{D}$ with $M \subseteq N \backslash\left(N_{x}^{G}(R) \cup N_{x}^{B}(R)\right)$,

$$
S \subseteq N_{x}^{B}(R) \Rightarrow x \notin \varphi(R) .
$$

When $M=\varnothing$, we say $S$ is negatively decisive for $\boldsymbol{x}$. A group $S \subseteq N \backslash M$ is negatively $M$-decisive if the group is negatively $M$-decisive for all objects. When $M=\varnothing$, we say $S$ is negatively decisive.

Moreover we also need to establish the following previous results, Lemmas 3-8. We omit the proof of the first one since it is straightforward.

Lemma 3. Let $\varphi$ be objectwise monotonic and $x \in A$. Let $M \subseteq N$. Assume that there exists $R \in \mathcal{D}$ such that $M \subseteq N \backslash\left(N_{x}^{G}(R) \cup N_{x}^{B}(R)\right)$ and $N_{x}^{G}(R)=$ $(N \backslash M) \backslash N_{x}^{B}(R)$.

(i) If $x \in \varphi(R)$, then $N_{x}^{G}(R)$ is positively $M$-decisive for $x$.

(ii) If $x \notin \varphi(R)$, then $N_{x}^{B}(R)$ is negatively $M$-decisive for $x$.

Lemma 4. Let $M \subseteq N$ and $N \backslash M \neq \varnothing$. Given an objectwise monotonic and efficient rule, if a group is positively $M$-decisive for an object, then the group is positively $M$-decisive.

Proof. We use Property $\mathrm{P}$ of the domain $\mathcal{D}$. The same proof can be established using Property $\mathrm{Q}$ instead. Let $M \subseteq N$ and $N \backslash M \neq \varnothing$. Let $\varphi$ be objectwise monotonic and efficient. Let $S \subseteq N \backslash M$ and $x \in A$. Assume that $S$ is positively $M$-decisive for $x$. Let $y \in A \backslash\{x\}$. We only have to show that $S$ is positively $M$-decisive for $y$.

For all $i \in S$, by $\mathrm{P} 1$, there exists $R_{i} \in \mathcal{D}_{i}$ such that

$$
\text { y } P_{i} x P_{i} \varnothing
$$

for all $z \in A \backslash\{x, y\}$,

$$
\varnothing P_{i} z \text {. }
$$

For all $j \in(N \backslash M) \backslash S$, by P1, there exists $R_{j} \in \mathcal{D}_{j}$ such that

$$
\varnothing P_{j} y P_{j} x,
$$

for all $z \in A \backslash\{x, y\}$,

$$
\varnothing P_{j} z .
$$

For all $m \in M$, by $\mathrm{P} 2$, there exists $R_{m} \in \mathcal{D}_{m}$ such that

$$
x I_{m} y I_{m} \varnothing,
$$


for all $z \in A \backslash\{x, y\}$,

$$
\varnothing P_{m} z .
$$

Then by efficiency, $\varphi(R) \subseteq\{x, y\}$. Since $S$ is positively $M$-decisive for $x$, then $\varphi(R)=\{x\}$ or $\{x, y\}$. Since for all $j \in N \backslash M,\{y\} P_{j}\{x\}$, for all $m \in M$, $\{y\} I_{m}\{x\}$, and $N \backslash M \neq \varnothing$, then by efficiency, $\varphi(R)=\{x, y\}$. Since $M \subseteq$ $N \backslash\left(N_{y}^{G}(R) \cup N_{y}^{B}(R)\right), N_{y}^{G}(R)=S$ and $N_{y}^{B}(R)=N \backslash S$, then, by Lemma $3, S$ is positively $M$-decisive for $y$.

Lemma 5. Let $M \subseteq N$ and $N \backslash M \neq \varnothing$. Given an objectwise monotonic and efficient rule, if a group containing more than two agents is positively $M$-decisive, then the group contains a positively $M$-decisive proper subgroup.

Proof. We use Property $\mathrm{P}$ of the domain $\mathcal{D}$. The same proof can be established using Property Q instead. Let $M \subseteq N$ and $N \backslash M \neq \varnothing$. Let $\varphi$ be objectwise monotonic and efficient. Let $S \subseteq N \backslash M,|S| \geq 2$, and $i \in S$. Assume that $S$ is positively $M$-decisive. We show that either $S \backslash i$ or $i$ is positively $M$-decisive. By Lemma 4, we only have to show that there exists $x \in A$ such that either $S \backslash i$ or $i$ is positively $M$-decisive for $x \in A$. Since $|A| \geq 3$, there exist three objects, $x, y, z$, in $A$.

By P1, there exists $R_{i} \in \mathcal{D}_{i}$ such that:

$$
y P_{i}\{y, z\} P_{i} x P_{i} \varnothing P_{i} z,
$$

for all $w \in A \backslash\{x, y, z\}$,

$$
\varnothing P_{i} w
$$

For all $j \in S \backslash\{i\}$, by $\mathrm{P} 1$, there exists $R_{j} \in \mathcal{D}_{j}$ such that:

$$
z P_{j}\{y, z\} P_{j} x P_{j} \varnothing P_{j} y
$$

for all $w \in A \backslash\{x, y, z\}$,

$$
\varnothing P_{j}\{w\} .
$$

For all $h \in(N \backslash M) \backslash S$, by P1, there exists $R_{h} \in \mathcal{D}_{h}$ such that:

$$
\begin{aligned}
& \varnothing P_{h}\{y\}, \\
& \varnothing P_{h}\{z\}, \\
& \varnothing P_{h}\{y, z\} P_{i}\{x\},
\end{aligned}
$$

for all $w \in A \backslash\{x, y, z\}$,

$$
\varnothing P_{h}\{w\}
$$


For all $m \in M$, by $\mathrm{P} 2$, there exists $R_{m} \in \mathcal{D}_{m}$ such that

$$
\{x\} I_{m}\{y\} I_{m}\{z\} I_{m} \varnothing
$$

for all $w \in A \backslash\{x, y, z\}$,

$$
\varnothing P_{m}\{w\} \text {. }
$$

Then by separability and efficiency, $\varphi(R) \subseteq\{x, y, z\}$. Since $S$ is positively $M$ decisive, then $x \in \varphi(R)$. Since for all $j \in N \backslash M,\{y, z\} P_{j}\{x\}$, for all $m \in M$, $\{y, z\} I_{m}\{x\}$, and $N \backslash M \neq \varnothing$, then by efficiency, $\varphi(R) \cap\{y, z\} \neq \varnothing$. Note that $M \subseteq N \backslash\left(N_{y}^{G}(R) \cup N_{y}^{B}(R)\right), N_{y}^{G}(R)=\{i\}, N_{y}^{B}(R)=(N \backslash M) \backslash\{i\}, M \subseteq$ $N \backslash\left(N_{z}^{G}(R) \cup N_{z}^{B}(R)\right), N_{z}^{G}(R)=S \backslash\{i\}$, and $N_{z}^{B}(R)=(N \backslash M) \backslash(S \backslash\{i\})$. Therefore by Lemma 3, if $y \in \varphi(R)$, then $i$ is positively $M$-decisive for $y$ and if $z \in \varphi(R)$, then $S \backslash\{i\}$ is positively $M$-decisive for $z$.

We next state two lemmas on negative decisiveness, corresponding to Lemmas 4 and 5 . The proofs are similar to the previous proofs.

Lemma 6. Let $M \subseteq N$ and $N \backslash M \neq \varnothing$. Given an objectwise monotonic and efficient rule, if a nonempty group is negatively $M$-decisive for an object, then the group is negatively $M$-decisive.

Lemma 7. Let $M \subseteq N$ and $N \backslash M \neq \varnothing$. Given an objectwise monotonic and efficient rule, if a group containing more than two agents is negatively $M$-decisive, then the group contains a negatively $M$-decisive proper subgroup.

For all $R_{i} \in \mathcal{D}_{i}$ and all $Y \subseteq A$, let $G\left(R_{i}, Y\right) \equiv G\left(R_{i}\right) \cap Y$ and $B\left(R_{i}, Y\right) \equiv$ $B\left(R_{i}\right) \cap Y$. We next state a useful property of a separable preference. The proof is trivial.

Lemma 8. Let $X$ and $Y$ be disjoint subsets of $A$. Let $R_{0} \in \mathcal{S}$. Then $T \in \operatorname{Max}\left\{R_{0},\{X \cup Z: Z \subseteq Y\}\right\} \Leftrightarrow X \cup G\left(R_{0}, Y\right) \subseteq T \subseteq X \cup\left(Y \backslash B\left(R_{0}, Y\right)\right)$.

Lemma 9. If a rule is objectwise monotonic and efficient, then it is serially dictatorial.

Proof. Let $\varphi$ be objectwise monotonic and efficient.

Step 1. There exists a permutation $\pi$ on $N$ such that for all $k \in\{1, \cdots, n\}$, $\pi(k)$ is $\{\pi(1), \cdots, \pi(k-1)\}$-decisive.

Let $M^{1} \equiv \varnothing$. We first show that there exists an agent who is both positively and negatively $M^{1}$-decisive. By efficiency of $\varphi, N$ is positively $M^{1}$-decisive. By Lemma 5 , there exists a positively $M^{1}$-decisive proper subgroup $S$ of $N$. If $S$ is 
singleton, then we are done. Otherwise we apply Lemma 5 to $S$. Since there are finite number of agents, then iterating this argument, we can find an agent $i \in N$ who is positively $M^{1}$-decisive. Using Lemma 7 and the same argument as above, we show that there exists a negatively $M^{1}$-decisive agent $j \in N$. If $i \neq j$, then for all $R \in \mathcal{D}$ with $a \in G\left(R_{i}\right)$ and $a \in B\left(R_{j}\right)$, we have $a \in \varphi(R)$ and $a \notin \varphi(R)$. This is a contradiction. Therefore $i=j$.

Let $i_{1}$ be $M^{1}$-decisive. Now let $M^{2} \equiv\left\{i_{1}\right\}$. If $N \backslash M^{2}$ is not empty, then by efficiency, $N \backslash M^{2}$ is $M^{2}$-decisive. Using the same argument as above, we show that there exists $i_{2} \in N \backslash M^{2}$ who is $M^{2}$-decisive. Let $M^{3} \equiv\left\{i_{1}, i_{2}\right\}$. Then if $N \backslash M^{3}$ is not empty, then by efficiency, $N \backslash M^{3}$ is $M^{3}$-decisive. Using the same argument as above, we show that there exists $i_{3} \in N \backslash M^{3}$ who is $M^{3}$-decisive. Proceeding this way, we find $\left(i_{1}, i_{2}, \cdots, i_{n}\right)$ such that for all $k \in\{1, \cdots, n\}, i_{k}$ is $M^{k}$-decisive, where $M^{k} \equiv\left\{i_{1}, \cdots, i_{k-1}\right\}$. Let $\pi$ be the permutation on $N$ such that for all $k \in N, \pi(k) \equiv i_{k}$. Then $\pi$ satisfies the desired property.

Step 2. Let $X_{\pi(1)} \equiv \varnothing$ and $Y_{\pi(1)} \equiv$ A. For all $k \in\{2, \cdots, n\}$, let $X_{\pi(k)} \equiv X_{\pi(k-1)} \cup G\left(R_{\pi(k-1)}, Y_{\pi(k-1)}\right)$ and $Y_{\pi(k)} \equiv Y_{\pi(k-1)} \backslash\left[G\left(R_{\pi(k-1)}, Y_{\pi(k-1)}\right) \cup B\left(R_{\pi(k-1)}, Y_{\pi(k-1)}\right)\right]$. Then for all $k \in\{1, \cdots, n-1\}, M^{k}(R, \pi)=\left\{X_{\pi(k+1)} \cup Z: Z \subseteq Y_{\pi(k+1)}\right\}$

Without loss of generality, we assume that $\pi(1)=1, \cdots, \pi(n)=n$. Clearly by separability, $M^{1}(R, \pi) \equiv \operatorname{Max}\left\{R_{1}, 2^{A}\right\}=\left\{G\left(R_{1}, A\right) \cup Z: Z \subseteq A \backslash\left[G\left(R_{1}, A\right) \cup B\left(R_{1}, A\right)\right]\right\}$. Therefore the result holds for $k=1$. Suppose by induction that the result holds for $k=m$, where $m \in\{1, \cdots, n-2\}$. By definition, $M^{m}(R, \pi) \equiv \operatorname{Max}\left\{R_{m}, M^{m-1}(R, \pi)\right\}$. Since by the induction hypothesis, $M^{m-1}(R, \pi)=\left\{X_{m} \cup Z: Z \subseteq Y_{m}\right\}$, then by Lemma 8, $M^{m}(R, \pi)=\left\{X_{m} \cup G\left(R_{m}, Y_{m}\right) \cup Z: Z \subseteq Y_{m} \backslash\left[G\left(R_{m}, Y_{m}\right) \cup B\left(R_{m}, Y_{m}\right)\right]\right.$. Hence $M^{m}(R, \pi)=\left\{X_{m+1} \cup Z: Z \subseteq Y_{m+1}\right\}$.

Step 3. The rule $\varphi$ is serially dictatorial with respect to $\pi$.

Without loss of generality, we assume that $\pi(1)=1, \cdots, \pi(n)=n$. By Step 2 and Lemma 8, we only have to show that for all $k \in N$,

$$
X_{k} \cup G\left(R_{k}, Y_{k}\right) \subseteq \varphi(R) \subseteq X_{k} \cup\left(Y_{k} \backslash B\left(R_{k}, Y_{k}\right)\right)
$$

Since agent 1 is decisive, $G\left(R_{1}\right) \subseteq \varphi(R) \subseteq A \backslash B\left(R_{1}\right)$. Hence (3) holds for $k=$ 1. Suppose by induction that (3) holds for $k \in\{2, \cdots, n-1\}$. We first show $X_{k+1} \cup G\left(R_{k+1}, Y_{k+1}\right) \subseteq \varphi(R)$. Let $x \in X_{k+1} \cup G\left(R_{k+1}, Y_{k+1}\right)$. If $x \in X_{k+1}$, then $x \in X_{k} \cup G\left(R_{k}, Y_{k}\right)$ and so by the induction hypothesis, $x \in \varphi(R)$. Let $x \in G\left(R_{k+1}, Y_{k+1}\right) \backslash X_{k+1}$. Since $x \in Y_{k+1}$, then for all $i \leq k,\{x\} I_{i} \varnothing$. Since $k+1$ is $\{1, \cdots, k\}$-decisive, $x \in \varphi(R)$. Therefore $X_{k+1} \cup G\left(R_{k+1}, Y_{k+1}\right) \subseteq \varphi(R)$. 
Next we show $\varphi(R) \subseteq X_{k+1} \cup\left(Y_{k+1} \backslash B\left(R_{k+1}, Y_{k+1}\right)\right)$. Suppose by contradiction that $x \notin X_{k+1} \cup\left(Y_{k+1} \backslash B\left(R_{k+1}, Y_{k+1}\right)\right)$. Then $x \notin X_{k+1}$. And $x \notin Y_{k+1}$ or $x \in$ $B\left(R_{k+1}, Y_{k+1}\right)$. In order to show $x \notin \varphi(R)$, we divide two cases.

Case 1. $x \notin X_{k+1}$ and $x \notin Y_{k+1}$. Since $x \notin X_{k+1}$, then for all $i \in\{1, \cdots, k\}$, $x \notin G\left(R_{i}, Y_{i}\right)$. Since $x \notin Y_{k+1}, x \in \cup_{i=1}^{k}\left[G\left(R_{i}, Y_{i}\right) \cup B\left(R_{i}, Y_{i}\right)\right]$. Therefore there exists $i^{*} \in\{1, \cdots, k\}$ such that $x \in B\left(R_{i^{*}}, Y_{i^{*}}\right)$ and for all $i<i^{*}, x \notin G\left(R_{i}, Y_{i}\right) \cup$ $B\left(R_{i}, Y_{i}\right)$. Clearly $x \in Y_{i^{*}}$. Since $Y_{1} \supseteq Y_{2} \supseteq \cdots \supseteq Y_{n}$, then for all $i<i^{*}$, $x \in Y_{i}$. Therefore for all $i<i^{*},\{x\} I_{i} \varnothing$. Since $i^{*}$ is $\left\{1, \cdots, i^{*}-1\right\}$-decisive and $x \in B\left(R_{i^{*}}, Y_{i^{*}}\right)$, then $x \notin \varphi(R)$.

Case 2. $x \notin X_{k+1}$ and $x \in B\left(R_{k+1}, Y_{k+1}\right)$. Since $x \in Y_{k+1}$, then for all $i \leq k,\{x\} I_{i} \varnothing$. Since $k+1$ is $\{1, \cdots, k\}$-decisive and $x \in B\left(R_{k+1}, Y_{k+1}\right)$, then $x \notin \varphi(R)$.

Proof of Proposition 2. By Lemma 2, every monotonic and independent rule is objectwise monotonic. Therefore it follows directly from Proposition 1 and Lemma 9, that if a rule satisfies the two axioms and efficiency, then it is a serially dictatorial rule in Family $\Phi^{*}$.

Note that the proofs of Lemmas 4-7 are carried out in such a way that when $M \equiv \varnothing$, they are valid replacing efficiency with weak efficiency. Therefore, using Lemmas 4-7 and the argument in the first part (four paragraphs) of Step 1 in Proof of Lemma 9, we show that if a rule is objectwise monotonic and weakly efficient, then there exists a decisive agent.

Lemma 10. If a rule is objectwise monotonic and weakly efficient, then there exists $i \in N$ such that for all $R \in \mathcal{D}, G\left(R_{i}\right) \subseteq \varphi(R) \subseteq A \backslash B\left(R_{i}\right)$.

Proof of Proposition 3. The proof of Proposition 3 follows directly from Proposition 1 and Lemma 10.

To prove Proposition 4 and therefore Theorems 1 and 2, we need the following four lemmas.

Lemma 11. Let $\varphi$ be a strategy-proof voting rule. Let $R \in \mathcal{D}, i \in N$, and $x \in A$. Then if $x \in \varphi(R)$, then for all $R_{i}^{\prime} \in \mathcal{D}_{i}$ with $x \in G\left(R_{i}^{\prime}\right), x \in \varphi\left(R_{i}^{\prime}, R_{-i}\right){ }^{29}$

Proof. Let $\varphi$ satisfy strategy-proofness and votes-only property. Let $i \in N$ and $x \in A$. Let $R \in \mathcal{D}$ and $R_{i}^{\prime} \in \mathcal{D}_{i}$ be such that $x \in \varphi(R)$ and $x \in G\left(R_{i}^{\prime}\right)$. By $\mathrm{R} 1$, there exists $R_{i}^{*} \in \mathcal{D}_{i}$ be such that $\left(G\left(R_{i}^{*}\right), B\left(R_{i}^{*}\right)\right) \equiv\left(G\left(R_{i}^{\prime}\right), B\left(R_{i}^{\prime}\right)\right)$ and for

\footnotetext{
${ }^{29}$ Lemmas 11 and 12 are similar to the arguments used by Barberà, Sonnenschein, and Zhou (1991) in their proof of Lemma 1.
} 
all $X, X^{\prime} \subseteq A \backslash\{x\}, X \cup\{x\} P_{i}^{*} X^{\prime}$.Then by votes-only property, $\varphi\left(R_{i}^{*}, R_{-i}\right)=$ $\varphi\left(R_{i}^{\prime}, R_{-i}\right)$. By strategy-proofness, $x \in \varphi\left(R_{i}^{*}, R_{-i}\right)$. Therefore $x \in \varphi\left(R_{i}^{\prime}, R_{-i}\right)$.

Lemma 12. Let $\varphi$ be a strategy-proof voting rule. Let $R \in \mathcal{D}, i \in N$, and $x \in A$. Then if $x \notin \varphi(R)$, then for all $R_{i}^{\prime} \in \mathcal{D}_{i}$ with $x \in B\left(R_{i}^{\prime}\right), x \notin \varphi\left(R_{i}^{\prime}, R_{-i}\right)$.

Proof. Let $\varphi$ satisfy strategy-proofness and votes-only property. Let $i \in N$ and $x \in A$. Let $R \in \mathcal{D}$ and $R_{i}^{\prime} \in \mathcal{D}_{i}$ be such that $x \notin \varphi(R)$ and $x \in B\left(R_{i}^{\prime}\right)$. By R2, there exists $R_{i}^{*} \in \mathcal{D}_{i}$ be such that $\left(G\left(R_{i}^{*}\right), B\left(R_{i}^{*}\right)\right) \equiv\left(G\left(R_{i}^{\prime}\right), B\left(R_{i}^{\prime}\right)\right)$ and for all $X \subseteq A \backslash\{x\}, X P_{i}^{*} X \cup\{x\}$. Then by votes-only property, $\varphi\left(R_{i}^{*}, R_{-i}\right)=\varphi\left(R_{i}^{\prime}, R_{-i}\right)$. By strategy-proofness, $x \notin \varphi\left(R_{i}^{*}, R_{-i}\right)$. Therefore $x \notin \varphi\left(R_{i}^{\prime}, R_{-i}\right)$.

Using Lemmas 11 and 12, we can establish Lemmas 13 and 14 which are crucial to prove Proposition 4.

Lemma 13. Every voting rule satisfying strategy-proofness and null-independence satisfies monotonicity.

Proof. Let $\varphi$ satisfy strategy-proofness, votes-only property, and null-independence. Let $R, R^{\prime} \in \mathcal{D}$ be such that for all $i \in N, G\left(R_{i}\right) \subseteq G\left(R_{i}^{\prime}\right)$ and $B\left(R_{i}\right) \supseteq B\left(R_{i}^{\prime}\right)$. Let $x \in \varphi(R)$. Then if $x \in G\left(R_{1}\right)$, then since $x \in G\left(R_{1}^{\prime}\right)$, by Lemma 11, we have $x \in \varphi\left(R_{1}^{\prime}, R_{-1}\right)$. If $x \in B\left(R_{1}\right)$, then by Lemma $12, x \in \varphi\left(R_{1}^{\prime}, R_{-1}\right)$. If $x \notin G\left(R_{1}\right) \cup B\left(R_{1}\right)$, then there are two cases: $x \in G\left(R_{1}^{\prime}\right)$ or $x \notin G\left(R_{1}^{\prime}\right) \cup B\left(R_{1}^{\prime}\right)$. In the first case, by Lemma $11, x \in \varphi\left(R_{1}^{\prime}, R_{-1}\right)$. In the second case, by nullindependence, $x \in \varphi\left(R_{1}^{\prime}, R_{-1}\right)$. Therefore, $\varphi(R) \subseteq \varphi\left(R_{1}^{\prime}, R_{-1}\right)$. Applying the same argument, successively changing preferences of agents, we can show that $\varphi(R) \subseteq \varphi\left(R^{\prime}\right)$.

Lemma 14. Every voting rule satisfying strategy-proofness and null-independence satisfies independence.

Proof. Let $\varphi$ satisfy strategy-proofness, votes-only property, and null-independence. Let $R, R^{\prime} \in \mathcal{D}$ be such that $\left.\left.R\right|_{\{\{x\}, \varnothing\}} \equiv R^{\prime}\right|_{\{\{x\}, \varnothing\}\}}$. We only have to show that $x \in \varphi(R) \Rightarrow x \in \varphi\left(R^{\prime}\right)$. Let $S_{1} \equiv N_{x}^{G}(R)\left(=N_{x}^{G}\left(R^{\prime}\right)\right), S_{2} \equiv N_{x}^{B}(R)\left(=N_{x}^{B}\left(R^{\prime}\right)\right)$, and $S_{3} \equiv N \backslash\left(S_{1} \cup S_{2}\right)$. For all $i \in S_{1}$, by Lemma 11, $x \in \varphi\left(R_{i}^{\prime}, R_{-i}\right)$. If we apply the same argument, changing preferences of agents in $S_{1}$ successively, then we obtain $x \in \varphi\left(R_{S_{1}}^{\prime}, R_{-S_{1}}\right)$. Let $\bar{R} \equiv\left(R_{S_{1}}^{\prime}, R_{-S_{1}}\right)$. For all $i \in S_{2}$, by Lemma 12 , $x \in \varphi\left(R_{i}^{\prime}, \bar{R}_{-i}\right)$. If we apply the same argument, changing preferences of agents in $S_{2}$ successively, then we obtain $x \in \varphi\left(R_{S_{2}}^{\prime}, \bar{R}_{-S_{2}}\right)$. Let $\hat{R} \equiv\left(R_{S_{2}}^{\prime}, \bar{R}_{-S_{2}}\right)$. For all $i \in S_{3}$, by null-independence, $x \in \varphi\left(R_{i}^{\prime}, \hat{R}_{-i}\right)$. If we apply the same 
argument, changing preferences of agents in $S_{3}$ successively, then we obtain $x \in \varphi\left(R_{S_{3}}^{\prime}, \hat{R}_{-S_{3}}\right)$. Therefore, since $\left(R_{S_{3}}^{\prime}, \hat{R}_{-S_{3}}\right)=R^{\prime}, x \in \varphi\left(R^{\prime}\right)$.

Proof of Proposition 4. By Lemmas 13 and 14, strategy-proofness and null-independence imply monotonicity and independence. The converse also holds, since every monotonic and independent rule is in Family $\Phi^{*}$ and so satisfies strategy-proofness and null-independence.

\section{References}

[1] Aizerman, M.A. and F.T. Aleskerov (1986), "Voting operators in the space of choice functions", Mathematical Social Sciences 11:201-242

[2] Aleskerov, F.T. and J. Duggan (1993), "Functional voting operators: the non-monotonic case", Mathematical Social Sciences 26:175-201

[3] Arrow, K.J. (1964), Social choice and individual values, $2^{\text {nd }}$ edition, New York: John Wiley

[4] Barberà, S., F. Gül, and E. Stacchetti (1993) "Generalized median voter schemes and committees", Journal of Economic Theory 61:262-289

[5] Barberà, S., J. Massó, and A. Neme (1997), "Voting under Constraints", Journal of Economic Theory 76:298-321

[6] Barberà, S., J. Massó, and A. Neme (2000), "Voting by committees under constraints", Working Paper

[7] Barberà, S., H. Sonnenschein, and L. Zhou (1991), "Voting by committees", Econometrica 59(3):595-609

[8] Blau, J.H. (1957), "The existence of social welfare function", Econometrica 25(2):302-313

[9] Border, K. and J. Jordan (1983), "Straightforward elections, unanimity and phantom voters", Review of Economic Studies 50:153-170

[10] Ferejohn, J.A. and P.C. Fishburn (1979), "Representations of binary decision rules by generalized decisiveness structures", Journal of Economic Theory 21:28-45 
[11] Gibbard, A. (1973), "Manipulation of voting scheme: a general result", Econometrica 41:587-602

[12] Guha, A.S. (1972), "Neutrality, monotonicity, and the right of veto", Econometrica 40(5):821-826

[13] Inada, K. (1969), "The simple majority decision rule", Econometrica 37(3):490-506

[14] Ju, B.-G. (2002a), "A characterization of plurality-like decision based on non-manipulability, restricted efficiency, and anonymity", University of Rochester, Mimeo

[15] Ju, B.-G. (2002b), "Coalitionally strategy-proof voting rules for separable weak orderings", University of Kansas, Mimeo

[16] Ju, B.-G. (2002c), "Plurality, Pareto efficiency, and strategy-proofness", University of Kansas, Mimeo

[17] Kasher, A. and A. Rubinstein (1998), "On the question 'Who is a j', a social choice approach", Forthcoming in Logique et Analyse

[18] Le Breton, M. and A. Sen (1999), "Separable preferences, strategyproofness, and decomposability", Econometrica 67(3):605-628

[19] Le Breton, M. and A. Sen (1995), "Strategyproofness and decomposability: weak orderings", Discussion Papers in Economics No. 95-04, Indian Statistical Institute, Delhi Centre

[20] Le Breton, M. and J. Weymark (1999), "Strategy-proof social choice with continuous separable preferences", Journal of Mathematical Economics 32(1):47-85

[21] May, K.O. (1952), "A set of independent necessary and sufficient conditions for simple majority decision", Econometrica 20(4):680-684

[22] Moulin, H. (1980), "On strategy-proofness and single-peakedness", Public Choice 35:435-455

[23] Moulin, H. (1993), "On the fair and coalition-strategyproof allocation of private goods", in Frontiers of Game Theory, edited by K. Binmore, A. Kirman, and P. Tani. The MIT Press, Cambridge 
[24] Murakami, Y. (1966), "Formal structure of majority decision", Econometrica 34(3):709-718

[25] Rubinstein, A. and P.C. Fishburn (1986), "Algebraic aggregation theory", Journal of Economic Theory 38:63-77

[26] Samet, D. and D. Schmeidler (2001), "Between liberalism and democracy", forthcoming in Journal of Economic Theory

[27] Satterthwaite, M. (1975), "Strategy-proofness and Arrow's conditions: existence and correspondence theorems for voting procedures and social welfare functions", Journal of Economic Theory 10:187-217

[28] Sen, A.K. (1977), "Social choice theory: a re-examination", Econometrica 45(1):53-89

[29] Serizawa, S. (1995), "Power of voters and domain of preferences where voting by committees is strategy-proof", Journal of Economic Theory 67:599-608

[30] Thomson, W. (2000), "Strategy-proof allocation rules on economic domains", University of Rochester, mimeo 